Partition function zeros for the Ising model on complete graphs and on annealed scale-free networks

This content has been downloaded from IOPscience. Please scroll down to see the full text.

2016 J. Phys. A: Math. Theor. 49135001

(http://iopscience.iop.org/1751-8121/49/13/135001)

View the table of contents for this issue, or go to the journal homepage for more

Download details:

IP Address: 193.50.135.4

This content was downloaded on 18/02/2016 at $16: 18$

Please note that terms and conditions apply. 


\title{
Partition function zeros for the Ising model on complete graphs and on annealed scale- free networks
}

\author{
M Krasnytska ${ }^{1,2,4,5}$, B Berche ${ }^{2,4}$, Yu Holovatch ${ }^{1,4}$ and \\ R Kenna ${ }^{3,4}$ \\ ${ }^{1}$ Institute for Condensed Matter Physics, National Acad. Sci. of Ukraine, UA-79011 \\ Lviv, Ukraine \\ ${ }^{2}$ Institut Jean Lamour, CNRS/UMR 7198, Groupe de Physique Statistique, Université \\ de Lorraine, BP 70239, F-54506 Vandœuvre-les-Nancy Cedex, France \\ ${ }^{3}$ Applied Mathematics Research Centre, Coventry University, Coventry CV1 5FB, UK \\ ${ }^{4}$ Doctoral College for the Statistical Physics of Complex Systems, Leipzig-Lorraine- \\ Lviv-Coventry $\left(\mathbb{L}^{4}\right)$, UK
}

E-mail: kras_mariana@icmp.lviv.ua, bertrand.berche@univ-lorraine.fr, hol@icmp. lviv.ua and R.Kenna@coventry.ac.uk

Received 10 September 2015, revised 27 November 2015

Accepted for publication 14 December 2015

Published DD MM 0000

\begin{abstract}
We analyse the partition function of the Ising model on graphs of two different types: complete graphs, wherein all nodes are mutually linked and annealed scale-free networks for which the degree distribution decays as $P(k) \sim k^{-\lambda}$. We are interested in zeros of the partition function in the cases of complex temperature or complex external field (Fisher and Lee-Yang zeros respectively). For the model on an annealed scale-free network, we find an integral representation for the partition function which, in the case $\lambda>5$, reproduces the zeros for the Ising model on a complete graph. For $3<\lambda<5$ we derive the $\lambda$-dependent angle at which the Fisher zeros impact onto the real temperature axis. This, in turn, gives access to the $\lambda$-dependent universal values of the critical exponents and critical amplitudes ratios. Our analysis of the Lee-Yang zeros reveals a difference in their behaviour for the Ising model on a complete graph and on an annealed scale-free network when $3<\lambda<5$. Whereas in the former case the zeros are purely imaginary, they have a non zero real part in latter case, so that the celebrated Lee-Yang circle theorem is violated.
\end{abstract}

Keywords: partition function zeros, phase transitions, Ising model, complex networks

5 Author to whom any correspondence should be addressed. 
(Some figures may appear in colour only in the online journal)

\section{Introduction}

Since the pioneering works of Lee and Yang [1], as well as Fisher [2], analysis of partition function zeros in the complex plane has become a standard tool to study properties of phase transitions in various systems [3], lattice spin models being one of them.

The Lee-Yang zeros are calculated at (real) temperature $(T)$ in the complex magnetic field $(H)$ plane whereas Fisher zeros (usually studied in the absence of a magnetic field) are located in the complex temperature plane. In the thermodynamic limit when the system size $N$ approaches infinity, the Lee-Yang and Fisher zeros form curves on the complex $(H$ or $T)$ plane. By analysing the location and scaling of these zeros, an alternative description of critical phenomena is achieved involving angles formed by these curves [4-8]. In this way the angles may be considered to be conjugate to the set of critical exponents and critical amplitudes ratios. Of special interest are the finite-size scaling (FSS) properties of zeros, which also encode universal features of underlying phase transitions [5].

Besides being of fundamental interest, and being useful for theory, the zeroes attract attention due to their experimental observation too. The first step to connect zeros to experimental data was made in [9]. The density of zeros on the Lee-Yang circle was determined by analysing isothermal magnetization data of the Ising ferromagnets. Recently Peng et al pointed out to possible relation between imaginary magnetic fields and quantum coherence of probe spin in a bath $[10,11]$. In turn, the experimentally obtained times at which quantum coherence disappears are equivalent to imaginary Lee-Yang zeros. Such demonstration of experimental realization of Lee-Yang zeros is important at a fundamental level and points to new ways of studying zeros in complex, many-bodied materials. E.g., the approach could help in the study of real systems in which the zeros can't easily be calculated giving access to new quantum phenomena that would otherwise remain hidden if one were to restrict attention to real, physical parameters. The experiments also confirm a profound connection between a static entity (the complex magnetic field in thermodynamics) and dynamical properties of quantum systems, namely coherence.

The aim of our paper is to analyse partition function zeros for a spin model on a complex network [12]. In particular, we are interested in a scale-free network with power-law decay of a node degree distribution $P(K) \sim K^{-\lambda}$, where $P(K)$ is the probability that network node has degree $K$. Such networks attract much attention due to their applications in descriptions of numerous natural and man-made systems, see e.g. [12, 13]. It is by now well established that models on such networks have qualitatively similar critical behaviour to those on systems defined on regular $d$-dimensional lattices. In particular, the exponent $\lambda$ determines collective behaviour and plays a role similar to that of the space dimension $d$ for lattice systems; there are lower and upper critical values of $\lambda$ for networks, analogous to lower and upper critical dimensions for lattices. At the upper critical values $\lambda=\lambda_{\mathrm{uc}}$ and $d=d_{\mathrm{uc}}$, scaling behaviour is modified by multiplicative logarithmic corrections, while above them, critical exponents assume their standard mean-field values. The above analogy has limitations; e.g., for $d \leqslant d_{\mathrm{lc}}$ lattice systems remain disordered at any finite temperature $T$ whereas systems on networks are always ordered at $\lambda \leqslant \lambda_{\mathrm{lc}}$. But the feature we want to emphasize by this comparison and which will be central for the analysis presented below is that $d$ and $\lambda$ play the role of global variables determining universal properties of critical behaviour. Therefore the parameter $\lambda$, like the dimensionality of a regular lattice, governs the universality of thermodynamic properties near the critical point along with the field symmetries and range of interactions. So, 
in the absence of a space dimensionality, $\lambda$ controls the transition. For the Ising model, $\lambda_{\mathrm{lc}}=3, \lambda_{\mathrm{uc}}=5$ while $d_{\mathrm{lc}}=1$ and $d_{\mathrm{uc}}=4$.

Here we consider the so-called annealed network (see e.g. [14]). For a spin model on such a network, the network configuration is fluctuating together with spins, which leads to the partition function configurational averaging [15]. In turn, this allows one to attain an asymptotically exact description of many spin models, placed on such a type of network [16]. The annealed network approximation gives the correct critical behaviour of the Ising model (and many others models [17]) on uncorrelated random complex networks. However, this approximation does not work when the network is close to the percolation point. In this description, the partition function is represented in the form of the model on a complete graph with random separable interactions [14]. Therefore, in our analysis we start from the Ising model on a complete graph [18, 19], considering properties of its partition function zeros in the complex $H$ and $T$ planes and then we will generalize this analysis for the case of an annealed scale-free network. The remainder of this paper is organized as follows. In section 2 we briefly introduce the main relations which follow from the properties of partition function zeros and fix the notations. Section 3 delivers the analysis of the Fisher and LeeYang zeros of the Ising model on a complete graph. Following [18] we represent the partition function in integral form, extracting the number of particles into the variable of integration and complex reduced temperature or magnetic field. In section 4 we obtain the results for both types of zeros for the Ising model on an annealed scale-free network with a power-law decay of the node degree distribution characterized by $\lambda$. We collect some details of our calculations in the appendices. Certain results of the section 4.3 have been previously announced in a Letter [20].

\section{Definitions and notations}

In this section, we fix the notation and recall the derivation of scaling relations required for the rest of the paper. We wish to consider the partition function either at the critical temperature $T=T_{\mathrm{c}}$ in the complex magnetic field or at $H=0$ in complex temperature. To investigate the former, we write $H=\operatorname{Re} H+\mathrm{i} \operatorname{Im} H$ and determine the Lee-Yang zeros $H=H_{j}$ of $Z\left(T_{\mathrm{c}}, H\right)$ by searching numerically for the intersection in the complex magneticfield plane of the curves $\operatorname{Re} Z\left(T_{\mathrm{c}}, H\right)=0$ and $\operatorname{Im} Z\left(T_{\mathrm{c}}, H\right)=0$.

For our considerations in the complex temperature plane we write $T=\operatorname{Re} T+i$ $\operatorname{Im} T=T_{\mathrm{c}}(1+t)$, where $T_{\mathrm{c}}$ is the (real) critical temperature in zero field and $t=\rho \mathrm{e}^{\mathrm{i} \Phi}$ ( $t=\left(T-T_{\mathrm{c}}\right) / T_{\mathrm{c}}$ ) parametrizes position in the complex plane relative to $T_{\mathrm{c}}$ [21]. To determine the Fisher zeros, we search for intersections of $\operatorname{Re} Z(T, 0)=0$ and $\operatorname{Im} Z(T, 0)=0$. These Fisher zeros accumulate in the vicinity of the critical point $T_{\mathrm{c}}$ along a line and tend to pinch the positive real axis with the angle $\Phi(t \rightarrow 0)=\varphi[4,8,21]$. Consider the locus of Fisher zeros in the complex temperature plane depicted in figure 1. Fisher zeros are represented as light discs (blue online), the black one being the critical point position. The critical temperature is real, so the critical point is located on the real axis. Applying a real magnetic field to the system, we observe that Fisher zeros move in the complex $T$ plane along a curve which defines an angle $\psi$ with the positive real axis [5].

A useful relation connects the impact angle $\varphi$ with the exponent $\alpha$ of the specific heat and with the specific heat universal amplitude ratio $A_{-} / A_{+}$, where $A_{+}, A_{-}$are scaling amplitudes at $t>0, t<0$ respectively. On the real axis in the thermodynamic limit, the singular part of the free energy in the vicinity of critical point in the absence of magnetic field can be written as $f \simeq F_{ \pm}|t|^{2-\alpha}$. In the high-temperature phase given by $t>0$, this is 


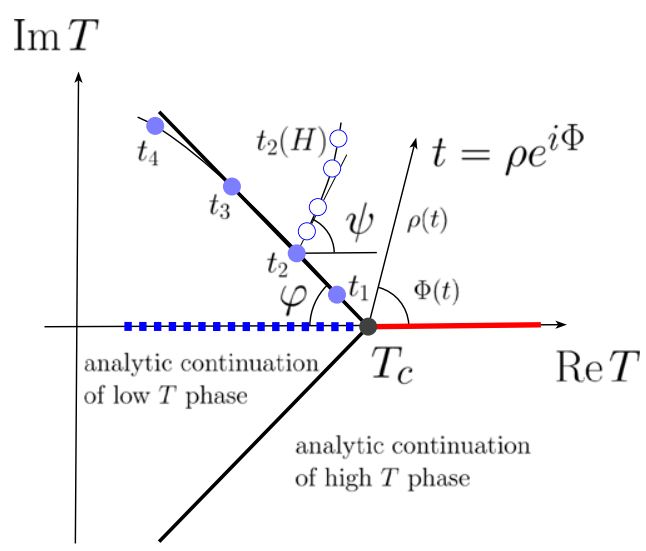

Figure 1. The distribution of Fisher zeros in the complex $T$ plane. The variable $t=\rho$ $\mathrm{e}^{\mathrm{i} \Phi}$ labels complex temperatures with respect to $T_{\mathrm{c}}$. The angle $\varphi$ is the impact angle of the zeros with the negative sense of the real axis, so that $\Phi \approx \pi-\varphi$ for the first few zeros which are indicated by light discs (blue online). The angle $\psi$ describes the motion of the Fisher zeros in presence of a real magnetic field. The motion of the first zero with increasing $H$ is indicated by the circles.

$f \simeq F_{+} t^{2-\alpha}$. In the low-temperature phase for which $t<0$ it is $f \simeq F_{-}(-t)^{2-\alpha}$. In the complex plane, the free energy is analytic everywhere except along the lines of zeros of the partition function. That line separates the two regions (high and low temperatures) in figure 1. By analytic continuation from the high temperature real axis, the region on the right of the line of zeros (the 'high temperature phase') has

$$
f_{+}(t) \simeq F_{+}\left(\rho \mathrm{e}^{\mathrm{i} \Phi}\right)^{2-\alpha}+f_{+}^{\mathrm{reg}}, \quad 0 \leqslant \Phi<\pi-\varphi .
$$

On the other hand, continuing the region to the left of the line of zeros (the low temperature phase), one has

$$
f_{-}(t) \simeq F_{-}\left(-\rho \mathrm{e}^{\mathrm{i} \Phi}\right)^{2-\alpha}+f_{-}^{\text {reg }}, \quad \pi-\varphi<\Phi \leqslant \pi .
$$

At the transition along the line of Fisher zeros, $\Phi=\pi-\varphi$, the real parts of the free energies of both phases are equal $[4,5]$. Substituting $\Phi=\pi-\varphi$ into (1), (2) and using the fact that $F_{-} / F_{+}=A_{-} / A_{+}$we arrive at the formula $[5,7,8]$ :

$$
\tan [(2-\alpha) \varphi]=\frac{\cos (\pi \alpha)-A_{-} / A_{+}}{\sin (\pi \alpha)} .
$$

The scaling of the zeros follows from general arguments. Replacing the volume $L^{d}$ of a regular lattice by the number of sites $N$ on a graph the partition function in terms of complex reduced temperature $t$ and magnetic field $H, Z(t, H)$, can be written as a generalized homogenous function [5]:

$$
Z(t, H)=Z\left(t N^{1 /(2-\alpha)}, H N^{\beta \delta /(2-\alpha)}\right) .
$$

The partition function is an even function of $H$ so that the previous scaling relation may be written either as

$$
H^{2} N^{2 \beta \delta /(2-\alpha)}=f\left(t N^{1 /(2-\alpha)}\right),
$$


or as

$$
t N^{1 /(2-\alpha)}=g\left(H N^{\beta \delta /(2-\alpha)}\right) .
$$

At $H=0$ equation (6) gives the scaling of Fisher zeros:

$$
t_{j}=N^{-1 /(2-\alpha)} g_{j}(0),
$$

where $g_{j}(0)$ is in general a complex number. Extending to scaling with the label index $j$ [5] we generalize from (5) and (6) to the Lee-Yang and Fisher zeros scaling as

$$
\begin{aligned}
& H_{j}(N, t=0) \sim\left(\frac{j}{N}\right)^{\frac{\beta \delta}{2-\alpha}}, \\
& t_{j}(N, H=0) \sim\left(\frac{j}{N}\right)^{\frac{1}{2-\alpha}} .
\end{aligned}
$$

Setting $t=0$ in (5) leads for the Lee-Yang zeros to [5]:

$$
H_{j}^{2}=N^{-2 \beta \delta /(2-\alpha)} f_{j}(0) \text {. }
$$

In general, $f_{j}(0)$ is a complex number. However, for models that obey the Lee-Yang theorem (all zeros are purely imaginary $H_{j} \sim \mathrm{i} \operatorname{Im} H_{j}$ [1]) $f_{j}(0)$ is a negative real number. Using this property one can derive from the scaling properties of the partition function [5] an angle $\psi$ of motion of Fisher zeros in real magnetic field. At fixed $t$ and for $N \rightarrow \infty$, the variable $H_{j}$ tends to the Lee-Yang edge, hence, substituting $N^{-1 /(2-\alpha)}$ from (7) into (10) we parametrize:

$$
t_{j} \sim H_{j}^{1 /(\beta \delta)} \exp ( \pm \mathrm{i} \psi)
$$

to obtain [5]

$$
\psi=\frac{\pi}{2 \beta \delta}
$$

\section{Ising model on a complete graph}

In this section we consider the infinite-range version of the Ising model which is equivalent to the mean-field version of its lattice counterpart [22, 23]. It can be also regarded as an Ising model on the complete graph, where each node of the graph is connected to every other node making all interspin couplings equal to each other. The analysis of the complex partition function zeros of this model which we present in this section serves two purposes. On the one hand, we complement the results of [18], obtained for Fisher zeros, by calculating the motion of these zeros in a real magnetic field and we use similar methodology to analyse Lee-Yang zeros. The Lee-Yang zeros for the exact partition function for the Ising model on a complete graph have been analysed previously [19], here we obtain the result for the expanded partition function and calculate coordinates of the Lee-Yang zeros in terms of the reduced variable, extracting dependency on the system size. On the other hand, we introduce the method which will be used in section 4 for a similar analysis of zeros for the model on an annealed network. The Hamiltonian of the model reads

$$
-\mathcal{H}=\frac{1}{2 N} \sum_{l \neq m} S_{l} S_{m}+H \sum_{l} S_{l} .
$$

Here, indices $l, m$ label the nodes of the graph so that $(l, m)=1, \ldots, N ; S_{l}= \pm 1$ are the Ising spins; $H$ is an external magnetic field and the sum $\sum_{l \neq m}$ spans all pairs of nodes (not 
necessarily the nearest neighbours). The coupling between the spins is taken to be inversely proportional to the number of nodes, to make the model meaningful in the thermodynamic limit $N \rightarrow \infty$. One can obtain an integral representation for the partition function of the model (13) by making use of the equality $\left(\sum_{l} S_{l}\right)^{2}=N+\sum_{l \neq m} S_{l} S_{m}$ to write for the $N$ particle partition function

$$
Z_{N}(T, H)=\operatorname{Tr} \mathrm{e}^{-\beta \mathcal{H}}=\mathrm{e}^{-\frac{\beta}{2}} \prod_{l=1}^{N} \sum_{S_{l}= \pm 1} \exp \left(\frac{\beta}{2 N}\left(\sum_{l} S_{l}\right)^{2}+\beta H \sum_{l} S_{l}\right),
$$

and then applying Hubbard-Stratonovich transformation to express the partition function in the form where summation over $S_{l}$ can be taken exactly:

$$
\begin{aligned}
& Z_{N}(T, H) \\
& =\mathrm{e}^{-\frac{\beta}{2}} \sqrt{\frac{N \beta}{2 \pi}} \prod_{l=1}^{N} \sum_{S_{l}= \pm 1} \int_{-\infty}^{+\infty} \exp \left(\frac{-N x^{2} \beta}{2}+\sum_{m} S_{m} \beta(x+H)\right) \mathrm{d} x .
\end{aligned}
$$

Performing the summation, one arrives at the integral representation for the partition function of the Ising model on the complete graph:

$$
Z_{N}(T, H)=\int_{-\infty}^{+\infty} \exp \left(\frac{-N x^{2}}{2 T}+N \ln \cosh [(x+H) / T]\right) \mathrm{d} x,
$$

where we have explicitly written the temperature $T=\beta^{-1}$ dependency taking the Boltzmann constant value $k_{\mathrm{B}}=1$. In (16) and in all other partition function integral representations below, we omit the irrelevant prefactors.

In classical settings, to get thermodynamic functions, the integral (16) is taken by the steepest descent method, see, e.g. [23]. In particular, the model undergoes a second order phase transition at $T_{\mathrm{c}}=1$ which is governed by the standard mean-field values of the critical exponents:

$$
\alpha=0, \quad \beta=1 / 2, \quad \delta=3, \quad \gamma=1 .
$$

For the sake of convenience it is appropriate to rewrite the partition function (16) in the reduced temperature variable $t=\left(T-T_{\mathrm{c}}\right) / T_{\mathrm{c}}=T-1$. Changing the integration variable $\sqrt{N} x / T \rightarrow x$ one gets [18]:

$Z_{N}(t, H)=\int_{-\infty}^{+\infty} \exp \left(\frac{-x^{2}(t+1)}{2}+N \ln \cosh [x / \sqrt{N}+H /(t+1)]\right) \mathrm{d} x$,

where the temperature dependent prefactor again has been omitted. Being primarily interested in the properties of the partition function itself, we approximate (18) by its expansion at large $N$ and small $H$. Keeping the leading order contributions in the linear in $H$ term, we expand the exponent function for $N \rightarrow \infty$ and get [18]:

$$
Z_{N}^{\exp }(t, H)=\int_{-\infty}^{+\infty} \exp \left(\frac{-t x^{2}}{2}-\frac{x^{4}}{12 N}+\frac{x \sqrt{N} H}{t+1}+O\left(1 / N^{2}\right)\right) \mathrm{d} x .
$$

In the remainder of this section we analyse the expressions for the exact and approximated partition functions of the Ising model on a complete graph, equations (18) and (19).

\subsection{Fisher zeros for the Ising model on a complete graph at $H=0$}

We start from the analysis of the exact integral representation for the partition function at zero external field. First, we obtain the Fisher zeros by solving the system of equations $\operatorname{Re} Z(t$, 

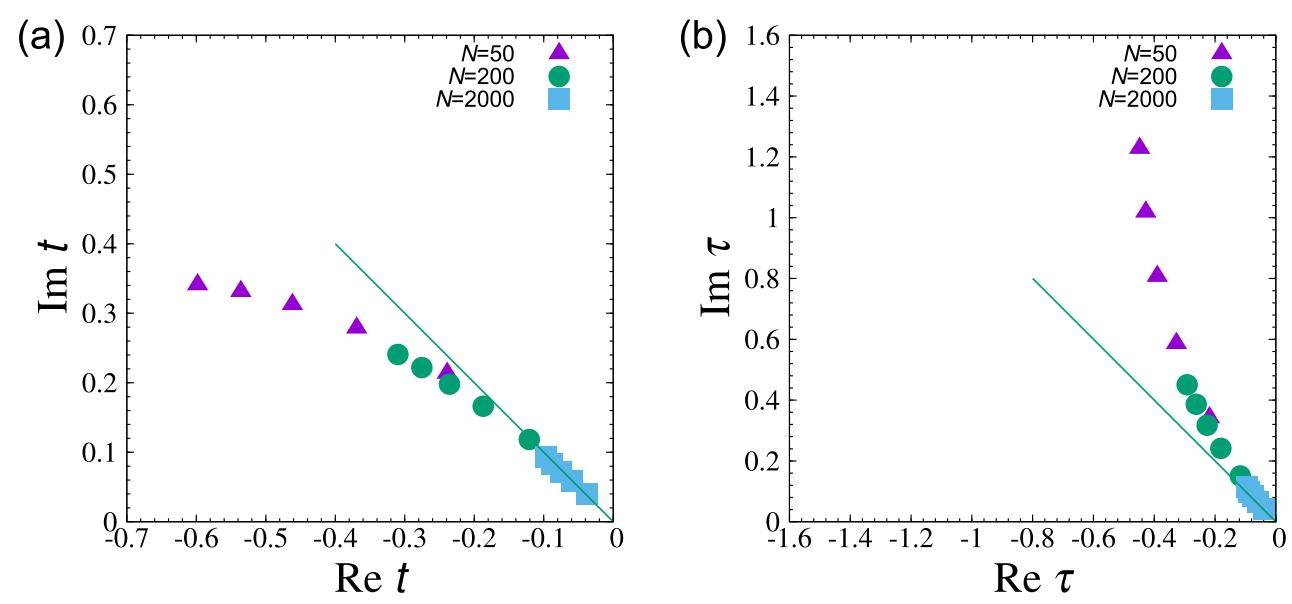

Figure 2. The first five Fisher zeros for the exact partition function (18) with different values of $N$ (a) in the complex $t$ plane $(t=T-1)$ and (b) in the complex $\tau$ plane $\tau=1-1 / T$. With increasing $N$, the zeros accumulate at the critical point, pinching the real axis at $t=\tau=0$. The solid line has an angle $\varphi=\pi / 4$ with the real axis.

$H)=0$ and $\operatorname{Im} Z(t, H)=0$ for the complex variables $t=\operatorname{Re} t+\mathrm{i} \operatorname{Im} t$ using function (18) at $H=0$. In figure 2(a) we show the first five Fisher zeros in the $t$ plane for increasing values of $N=50,200,2000$. They collapse on a master curve and in the vicinity of the critical point, impact onto the real axis, defining the angle $\varphi$.

It is instructive also to observe the motion of the zeros if the temperature is parameterized in a different way. Let us introduce the reduced inverse temperature:

$$
\left.\tau=\left(1 / T_{\mathrm{c}}-1 / T\right) /(1 / T)_{\mathrm{c}}\right)=1-1 / T,
$$

getting from (16) at $H=0$ :

$$
Z_{N}(\tau)=\int_{-\infty}^{+\infty} \exp \left(\frac{-N x^{2}(1-\tau)}{2}+N \ln \cosh [(1-\tau) x]\right) \mathrm{d} x .
$$

The zeros of the function (21) in the complex $\tau$ plane are shown in figure 2(b). As one can see from the figures, the zeros tend to form a smooth curve and accumulate in the vicinity of the critical point $(t=\tau=0)$, and with an increase of $N$ they tend to pinch the real positive temperature axis at the critical point. Using known values of the critical exponents and amplitude ratios one can evaluate the value of the angle $\varphi$ under which the zeros pinch the real axis. Substituting (17) into (3) and taking that the corresponding heat capacity amplitude ratio $A_{+} / A_{-}=0$ one arrives at

$$
\varphi=\pi / 4 \text {. }
$$

This value in shown in the figure 2 by the solid line. The fact that the lines along which the zeros tend to accumulate with increasing $N$ make the same angle $\varphi$ both in the $t$ and $\tau$ planes illustrates the conformal invariance of this angle - angles are independent of any analytic parametrization in the complex plane. However, the approach of the angle to its value in the thermodynamic limit $N \rightarrow \infty$ is parametrization dependent. Indeed, depending on parametrization, the curves of figure 2 reach the asymptotics from below (panel (a)) or from above (panel (b)). This is further outlined in figure 3, where we show how the values of the angle $\varphi$ and of the critical temperature change with $N$. To this end, we calculate the first five 

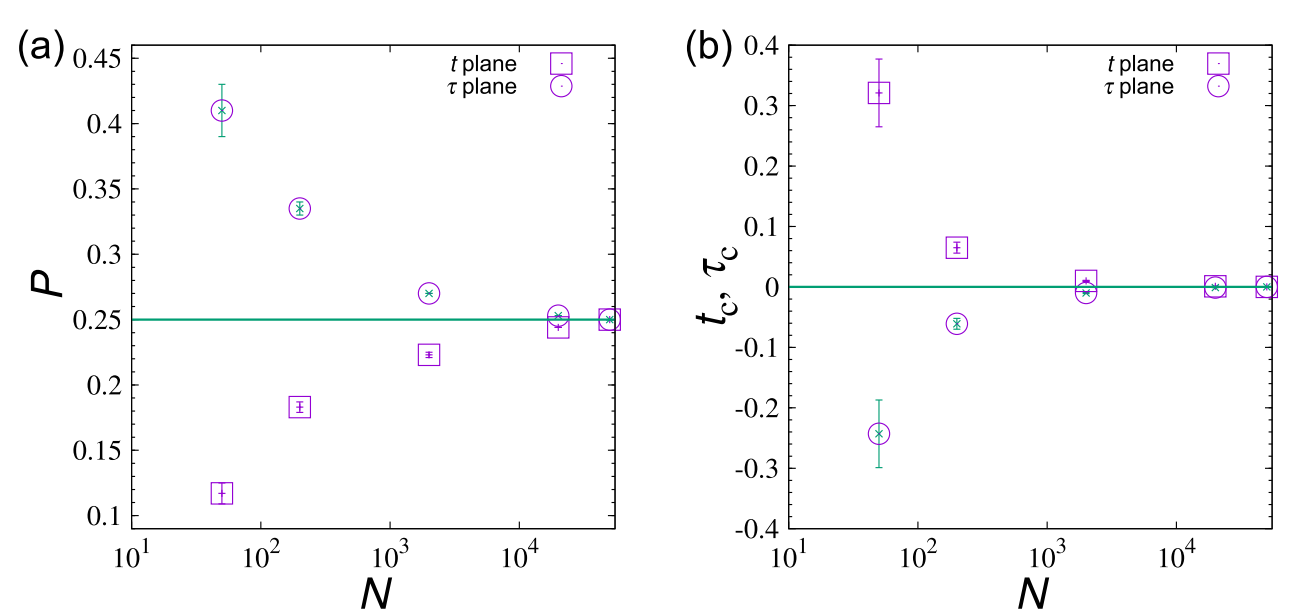

Figure 3. Values of (a) the reduced impact angle $P=\varphi / \pi$ and of (b) the critical temperature for the exact partition function obtained by fitting of the first five Fisher zeros for different $N$. Squares: results in the complex $t$ plane; discs: results in the complex $\tau$ plane; lines: exact results. The larger the system size, the more accurate is the fit. For $N>100$ the accuracy interval is less than the size of the data point in the figure.

Fisher zeros for the system sizes ranging up to $N=50000$, fit these points by a straight line and find the values of the angle (taking $P=\varphi / \pi$ so that equation (22) predicts $P=0.25$ ) and of the crossing point of this line with the real axis. The larger the system size, the more accurate the fit is (for $N>100$ the accuracy interval is less than the size of the data point in the figure). Starting from $N=5000, \varphi$ differs from its exact value $(\varphi=\pi / 4)$ by less than $1 \%$ while the critical temperature differs from the exact value at $t=0$ by less than $1 \%$ starting from $N=10000$.

Figures 2 and 3 quantify the main features of the behaviour of the Fisher zeros for the exact partition function (16) in the complex temperature plane. Results for the impact angle $\varphi$ and for the critical temperature obtained on their basis if compared with the exact results demonstrate two tendencies: (i) they improve with an increase of $N$ and (ii) the agreement is better for smaller values of the index $j$.

We now turn our attention to the zeros of the approximated partition function (19) to check how are these tendencies manifested in this case. For zero magnetic field, keeping only the term leading in $1 / N$, the partition function (19) reads

$$
Z_{N}^{\exp }(t)=\int_{-\infty}^{+\infty} \exp \left(-\frac{x^{2} t}{2}-\frac{x^{4}}{12 N}\right) \mathrm{d} x
$$

The first five Fisher zeros for the approximated partition function (23) are shown in the complex $t$ plane in figure 4(a) for different values of $N$. Similarly as for the exact partition function (see figure 2(a)), with an increase of $N$, the zeros accumulate in the vicinity of the critical point and tend to pinch the real axis at the critical point. An obvious difference in the behaviour of zeros for the exact and expanded partition functions is that in the last case the angle of zeros accumulation is very robust and almost does not depend on $N$. The reason becomes apparent when the function under the integral in (23) is rewritten as 

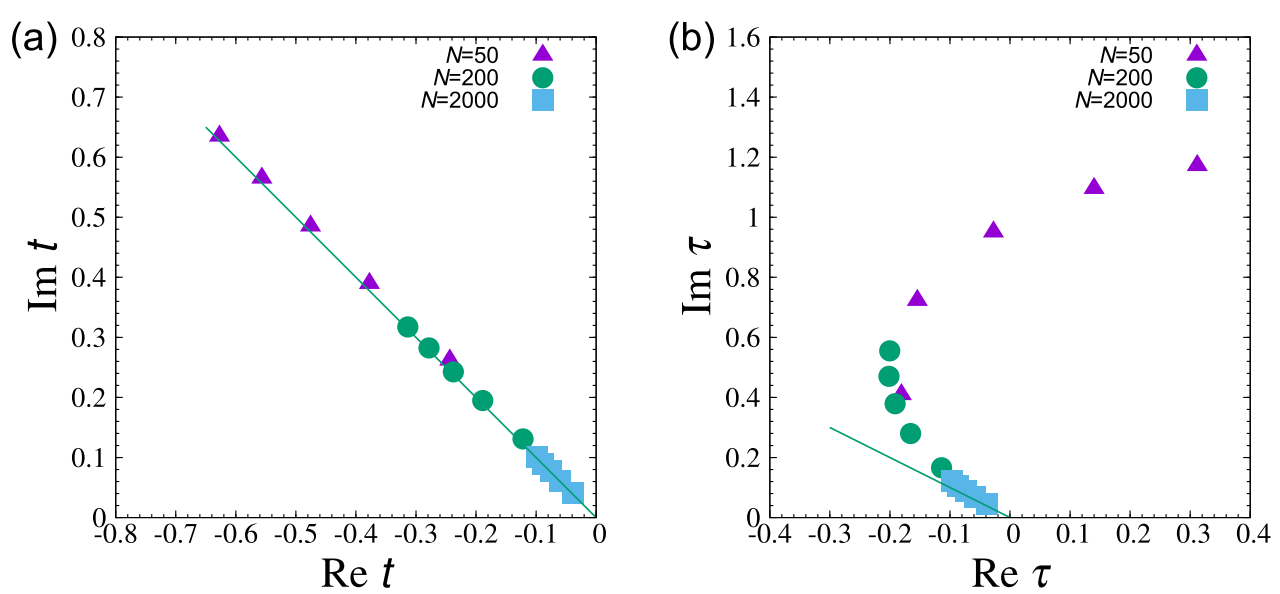

Figure 4. First five Fisher zeros for the approximated partition function for different values of $N$ (a) in the complex $t$ plane (equation (23)) and (b) in the complex $\tau$ plane (equation (26)). The solid line has an impact angle $\varphi=\pi / 4$.

$$
Z(z)=\int_{-\infty}^{+\infty} \exp \left(-z x^{2}-x^{4}\right) \mathrm{d} x
$$

where the $N$ and temperature dependencies are combined in the single scaling variable $z$

$$
z=\sqrt{3 N} t \text {. }
$$

Now it is easy to see that the connection between two sets of zeros $t_{j}\left(N_{1}\right)$ and $t_{j}\left(N_{2}\right)$ calculated for two different system sizes $N_{1}$ and $N_{2}$ is given by a simple rescaling: $t_{j}\left(N_{1}\right)=t_{j}\left(N_{2}\right) \sqrt{N_{1} / N_{2}}$. This, in turn, is manifested by the fact that zeros calculated for different system sizes align along the same line in figure 4(a).

The same expression (23), written for different values of $N$ expressed in terms of the $\tau$ variable takes the form

$$
Z_{N}^{\exp }(\tau)=\int_{-\infty}^{+\infty} \exp \left(-\frac{x^{2}\left(\tau-\tau^{2}\right)}{2}-\frac{x^{4}(1-\tau)^{4}}{12 N}\right) \mathrm{d} x
$$

Here, the $\tau$ dependence is nonlinear and the zeros are non-trivial functions of $N$. Indeed, the first few are depicted in figure 4(b) where one finds behaviour rather similar to that demonstrated in figure 2 for the exact partition function: sets of zeros calculated at different $N$ tend to form the common curved locus. However, evaluating (26) near the critical point for small $\tau$ and keeping the leading order contributions in the linear term in $\tau$, one arrives at the expression for $Z_{N}^{\exp }(\tau)$ that coincides with the corresponding expression (19) for $Z_{N}^{\exp }(t)$ in which $t$ is simply substituted by $\tau$. In turn, at zero magnetic field and close to the critical point, functions $Z_{N}^{\exp }(\tau)$ and $Z_{N}^{\exp }(t)$ attain the same form given by equation (23). In particular, in this approximation the $Z_{N}^{\exp }(\tau)$ function was considered in [18].

Zeros of the partition function (24) in the complex $z$ plane are shown in figure 5 . The thick and thin curves (green and violet online) give numerical solutions of the equations $\operatorname{Re} Z$ $(z)=0$ and $\operatorname{Im} Z(z)=0$, respectively. The crossing points of the lines give coordinates of the Fisher zeros. These have to align in the asymptotic limit $N \rightarrow \infty$ along the line forming an angle $\varphi=\pi / 4$ and starting at the critical point (the straight line in the figure). As it is clearly seen within the scale of the figure, the higher the index of the zero, the closer it is to the line. This tendency is further outlined in figure 6 , which demonstrates the behaviour of the angle $\varphi$ 


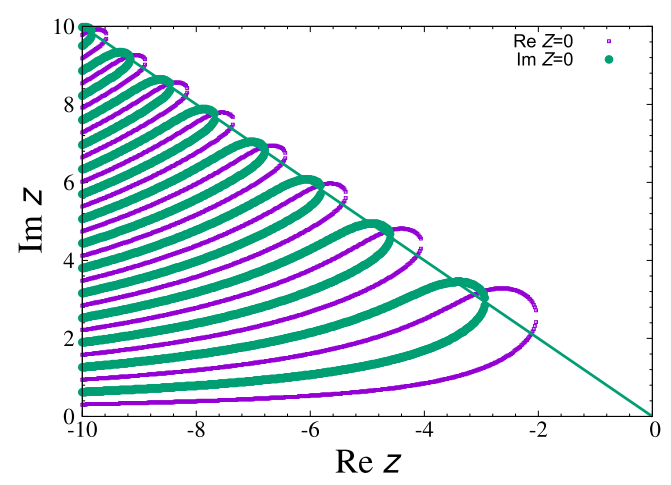

Figure 5. Loci of zeros for the real and imaginary parts of the approximated partition function (24) are plotted as thick and thin curves respectively (green and violet dots online). The points where the lines cross give the coordinates of the Fisher zeros. These have to align along the straight solid line in the asymptotic limit.
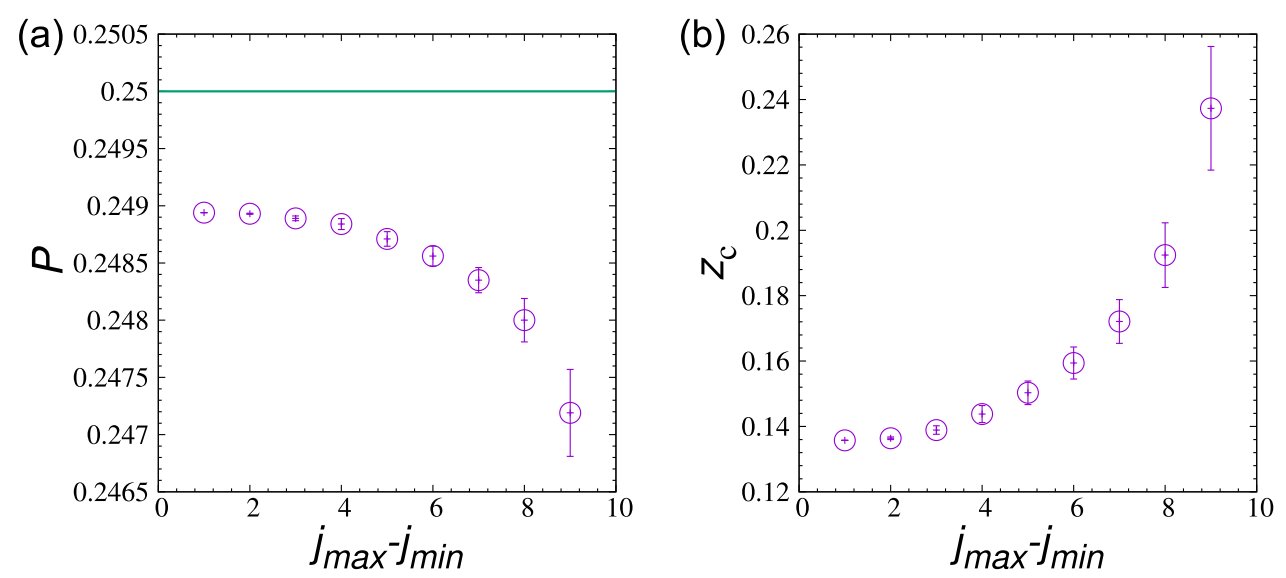

Figure 6. Estimates (a) for the reduced impact angle $P=\varphi / \pi$ and (b) for the critical temperature $z_{\mathrm{c}}$ for the partition function (24) obtained by fitting of Fisher zeros in the interval $j=j_{\min }, \ldots, j_{\max }$ for $j_{\max }=10$ and different values of $j_{\min }$. The solid line in panel (a) represents the exact value $P=1 / 4$.

and of the estimates for critical value $z_{\mathrm{c}}$ if they are obtained by fitting finite numbers of Fisher zeros in the interval $j=j_{\min }, \ldots, j_{\max }$ to a straight line. Fits for high $j$-values give better results. This tendency differs from the one observed above for the zeros in complex $t$ and $\tau$ planes (see figure 4) and is explained by the form of the scaling variable (25), which incorporates now both the temperature and the system size dependencies.

The coordinates of the first nine Fisher zeros in the $z$ plane as found numerically are listed in the left column of table 1 . As one observes from the table, as the zero-index $j$ increases, its real and imaginary part become closer, which corresponds to an approach of $\varphi$ to the value $\varphi=\pi / 4$ (see also figure 5). The value of $z_{j}$ for high $j$ can be evaluated asymptotically, writing the partition function (24) via the special functions: 
Table 1. Fisher zeros for the partition function (24). The second column shows our numerical results for the zeros given by $j=1, \ldots, 9$. In the third column they are compared with the values given by the asymptotic formula (28). When digits in the left and right columns coincide, they are underlined. Starting from $j=9$ the numerically calculated values coincide with their asymptotic counterparts within the accuracy presented.

\begin{tabular}{|c|c|c|}
\hline$j$ & Numeric & Equation (28) \\
\hline 1 & $-\underline{2.985} 52+\mathrm{i} \underline{3.2061}$ & $-\underline{2.98} 23+\mathrm{i} \underline{3.2023}$ \\
\hline 2 & $-\underline{4.623} 6+\mathrm{i} \overline{4.7707}$ & $-\underline{4.623} 1+i \underline{4.7700}$ \\
\hline 3 & $-\underline{5.8237}+\mathrm{i} \underline{5.9414}$ & $-\overline{5.823} 5+\mathrm{i} \underline{5.941} 1$ \\
\hline 4 & $-\underline{6.8167}+\mathrm{i} \underline{6.9176}$ & $-\underline{6.8167}+\mathrm{i} \underline{6.9174}$ \\
\hline 5 & $-\overline{7.6829}+\mathrm{i} \overline{7.7725}$ & $-\overline{7.6828}+\mathrm{i} \overline{7.7724}$ \\
\hline 6 & $-\underline{8.4610}+\underline{\mathrm{i} \underline{8.5425}}$ & $-\underline{8.4609}+\mathrm{i} \underline{8.542} 4$ \\
\hline 7 & $-\underline{9.1734}+\mathrm{i} \overline{9.2486}$ & $-\underline{9.173} 3+\mathrm{i} \overline{9.2486}$ \\
\hline 8 & $-\underline{9.8343}+\mathrm{i} \underline{9.9046}$ & $-\underline{9.8343}+\mathrm{i} 9.9045$ \\
\hline 9 & $-\underline{10.4536}+\mathrm{i} \underline{10.5197}$ & $-\underline{10.4536}+\mathrm{i} \underline{10.5197}$ \\
\hline
\end{tabular}

$$
Z(z)=\frac{\sqrt{z}}{4} \exp \left(\frac{z^{2}}{8}\right) K_{1 / 4}\left(\frac{z^{2}}{8}\right)=\frac{\sqrt{\pi}}{2^{5 / 4}} \exp \left(\frac{z^{2}}{8}\right) D_{-1 / 2}\left(\frac{z}{2^{1 / 2}}\right),
$$

where $K_{1 / 4}(x)$ and $D_{-1 / 2}(x)$ are the Bessel and parabolic cylinder functions [24], respectively. The leading term of the asymptotic expansion reads [18]:

$$
z_{j} \simeq 2(2 \pi j)^{1 / 2} \exp (3 \pi \mathrm{i} / 4)(1+O(1 / j))
$$

which in turn leads to the fact that $\operatorname{Re} z_{j}=-\operatorname{Im} z_{j}=\sqrt{4 \pi j}$ for high $j$. This asymptotic value is shown for different $j$ in the right column of table 1. Equation (28) has been obtained in [18] for $\left|z_{j}\right| \gg 1$. Since already for the first zero $\left|z_{1}\right| \simeq 4$, the asymptotic formula gives quite accurate values for the zeros for all $j$ as one may see comparing the left and right columns of the table. Starting from $j=9$ the numerically calculated values coincide with their asymptotic counterparts within the accuracy presented.

\subsection{Lee-Yang zeros for the Ising model on the complete graph at $T=T_{c}$}

We now turn to the analysis of the partition function for complex magnetic field $H$ at $T=T_{\mathrm{c}}$. The exact expression for the partition function (18) at $t=\tau=0$ reads:

$$
Z_{N}(H)=\int_{-\infty}^{+\infty} \exp \left(-\frac{x^{2}}{2}+N \ln \cosh [x / \sqrt{N}+H]\right) \mathrm{d} x .
$$

On the other hand, keeping only contributions leading in $N^{-1}$ in the expanded partition function (19) one gets:

$$
\left.Z_{N}^{\exp }(H)=\int_{-\infty}^{+\infty} \exp \left(-\frac{x^{4}}{12 N}+x \sqrt{N} H\right)\right) \mathrm{d} x .
$$

It has been suggested [5] that at the critical point the partition function zeros scale as a fraction of their total number $j / N$ for large values of the index $j$. Moreover, many models give scaling in the ratio $(j-C) / N$ in which $C=1 / 2$ is an empirical fitting factor [25, 26]. Recently, a more comprehensive form for the scaling of the Lee-Yang zeros in the critical region was suggested [27]. In our case it reads 

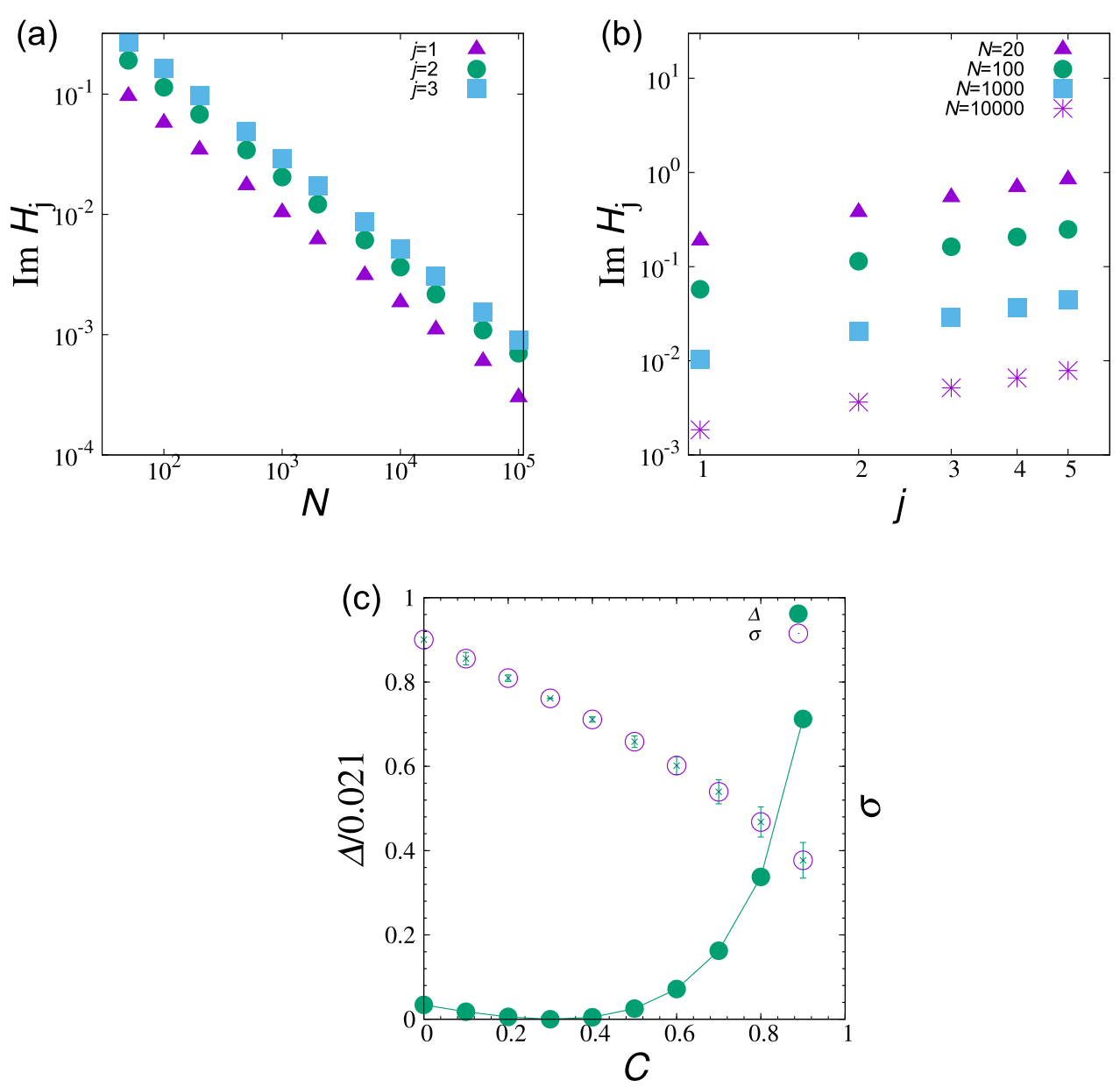

Figure 7. The behaviour of $\operatorname{Im} H_{j}$ for several leading Lee-Yang zeros of the exact partition function (29). (a) The finite-size $\operatorname{Im} H_{j}(N)$ dependency for first three zeros $j=1,2,3$. The scaling with $N$, equation (31), holds even for small $N$ and $j$ with an exponent very close to $\sigma=3 / 4$. (b) Coordinates of the first five zeros calculated for several values of $N=20,100,1000,10000$. The scaling exponents remain almost unchanged for different $N$ but they are far from their asymptotic value. (c) Variance of residuals $\Delta$ and exponent $\sigma$ obtained while fitting coordinates of the first five Lee Yang zeros via equation (31) for $N=1000$ as functions of the fitting parameter $C$. The optimal $\sigma$ is evaluated at $C_{\mathrm{opt}}=0.31$ where the $\Delta(C)$ curve has minimum. The resulting value $\sigma\left(C_{\text {opt }}\right)=0.7563(1)$ is close to the exact result $\sigma=0.75$.

$$
\operatorname{Im} H_{j} \sim\left(\frac{j-C}{N}\right)^{\sigma}
$$

where the exponent $\sigma$ is related to the order parameter and heat capacity critical exponents via

$$
\sigma=\frac{\beta \delta}{2-\alpha} \text {. }
$$




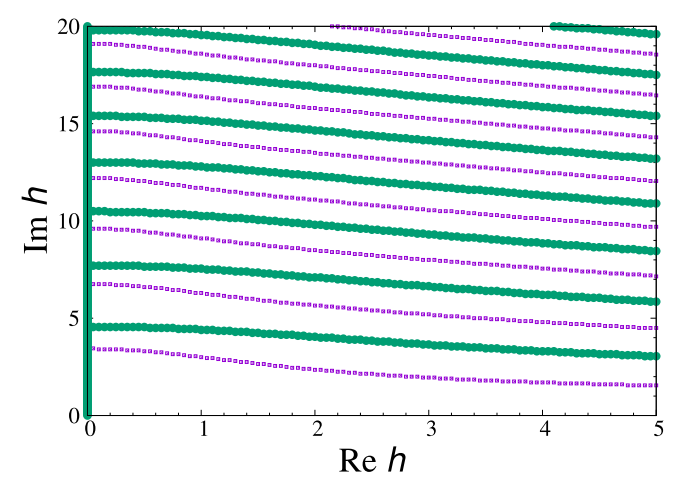

Figure 8. Lines of zeros for the real and imaginary part of the approximated partition function (34) at $T=T_{\mathrm{c}}$ in the complex magnetic field plane, thin and thick curves (violet and green online) respectively. The points where the lines of different colour cross give the coordinates of the Lee-Yang zeros. Note that one of the $\operatorname{Im} Z(h)=0$ lines coincides with the vertical axis in the plot.

Substituting the values of the exponents (17) into (32) one arrives at

$$
\sigma=3 / 4 \text {. }
$$

Next we check whether the scaling form (31) holds for the Lee-Yang zeros of the exact and approximated partition functions and we seek to estimate the value of the parameter $C$.

Numerically solving the system of equations $\operatorname{Re} Z(t, H)=0$ and $\operatorname{Im} Z(t, H)=0$ at $t=t_{\mathrm{c}}=0$ for the real and imaginary parts of the exact partition function (29) we get coordinates of its zeros in the complex $H$ plane. We find that the Lee-Yang zeros are purely imaginary in this case. The fact that they lie on the imaginary axis validates the famous LeeYang circle theorem [1]: expressed in terms of the variable $\mathrm{e}^{H}$ all zeros lie on a circle of unit radius. In figure 7(a) we plot numerical values of the coordinates of the first three Lee-Yang zeros as function of the system size $N$. There are two remarkable feature of the plots of figure 7(a): (i) all $H_{j}(N)$ dependencies are power laws (represented by straight lines in the log$\log$ plots); (ii) these power laws are governed by the same value of the exponent (the lines are parallel) for different $j$. Therefore, the scaling with $N$ as it is predicted by the equation (31) holds even for small $N$ and $j$. Using linear fits for all eleven data points of the figure 7(a) we find for each $j: \sigma=0.749(5)(j=1), \sigma=0.744(3)(j=2), \sigma=0.750(1)(j=3)$.

To check how the scaling of the zeros holds with $j$, we plot in figure 7(b) coordinates of the first five zeros calculated for several values of $N=20,100,1000,10000$. The fits to the power law dependency gives the following values of the exponent $\sigma=0.930(22)(N=20)$, $\sigma=0.909(22) \quad(N=100), \sigma=0.901(22) \quad(N=1000), \sigma=0.900(21) \quad(N=10000):$ the exponents remain almost unchanged for different $N$ but they are far from their asymptotic value $\sigma=3 / 4$, equation (33). However, introducing a fit parameter $C$ via equation (31) changes the picture.We fit the dependence of the first five Lee-Yang zeros on $j$ on a $\log -\log$ scale by a linear function at different values of the fitting parameter $C$ and evaluate goodness of fits by calculating variance of residuals (reduced $\chi^{2}$ ), i.e. the weighted sum-of-squares residuals divided by the number of degrees of freedom, further denoted by $\Delta$. The $\Delta(C)$ curve is shown in figure 7(c), the optimal value of the fitting parameter $C_{\text {opt }}$ corresponds to minimum of $\Delta$. In our case $C_{\text {opt }}=0.31$. Therefore, the value of the exponent $\sigma$ is calculated as $\sigma\left(C_{\text {opt }}\right)=0.7563(1)$ and is close to the exact result $\sigma=0.75$. 

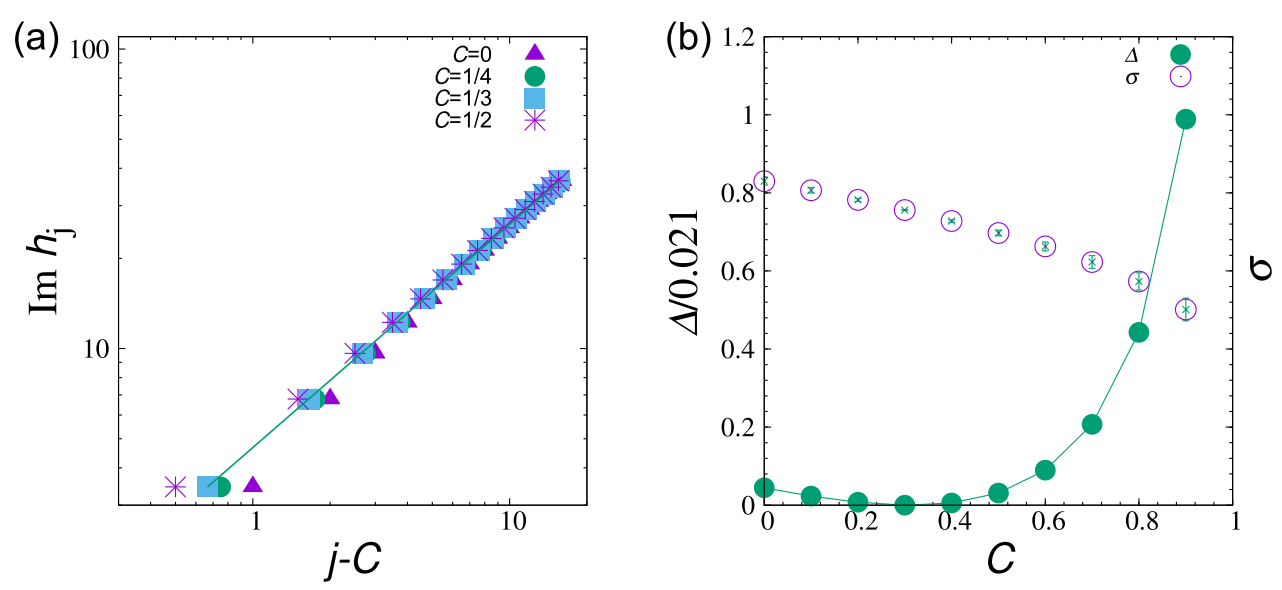

Figure 9. The behaviour of $\operatorname{Im} h_{j}$ for leading Lee-Yang zeros of the approximated partition function (34). (a) Coordinates $\operatorname{Im} h_{j}$ of numerically calculated first sixteen Lee-Yang zeros as functions of $j-C$ for different values of $C=0,1 / 4,1 / 3,1 / 2$. The solid line is an eye guide to show linear scaling with an exponent $\sigma=3 / 4$. Such scaling is manifested by a merge of the data points with an increase of $j$. (b) Variance of residuals $\Delta$ and exponent $\sigma$ obtained while fitting coordinates of the first sixteen Lee Yang zeros via equation (31) as functions of the fitting parameter $C$. The value of the exponent $\sigma\left(C_{\mathrm{opt}}\right)=0.7531(2)$ is close to the exact result $\sigma=0.75$.

For the expanded partition function, equation (30) can be conveniently written in terms of the rescaled variables:

$$
Z(h)=\int_{-\infty}^{+\infty} \exp \left(-x^{4}+x h\right) \mathrm{d} x,
$$

where the field and the system size dependence is absorbed into a single variable

$$
h=H\left(12 N^{3}\right)^{1 / 4} \text {. }
$$

Figure 8 displays the lines of zeros for the real and imaginary parts of the partition function (34) at $T=T_{\mathrm{c}}$ in the complex magnetic field $h$ plane, thin and thick curves (violet and green online) respectively. Again, the coordinates of the Lee-Yang zeros (the crossing points of the lines) are purely imaginary (obeying the Lee-Yang circle theorem). The scaling of $h_{j}$ with the system size $N$ is given by (35) and hence the scaling exponent is equal to its asymptotic value (33) for any index $j$. Note, that since the real part of the Lee-Yang zero coordinate $\operatorname{Re} h=0$, it is straightforward to show from (30) that the imaginary coordinate $\operatorname{Im} h$ is obtained as a solution of an integral equation:

$$
\int_{0}^{\infty} \mathrm{e}^{-x^{4}} \cos (x \operatorname{Im} h) \mathrm{d} x=0
$$

Let us consider now the scaling with $j$. To this end, in figure 9 we plot the numerically obtained coordinates $\operatorname{Im} h_{j}$ of the first sixteen Lee-Yang zeros as functions of $j-C$ for different values of $C=0,1 / 4,1 / 3,1 / 2$. As expected, for large enough $j$ the linear scaling with $j$ occurs and the data points align along a single line with the tangent $\sigma \simeq 3 / 4$. However, as for the exact partition function, this dependence is nonlinear for smaller values of $j$ and the choice of an appropriate fitting parameter $C$ similar as it was done for the exact function, see figure 7(c), allows to improve the linearity. As a result, we get the optimal value $C_{\mathrm{opt}}=0.31$ and $\sigma\left(C_{\text {opt }}\right)=0.7531(2)$. 

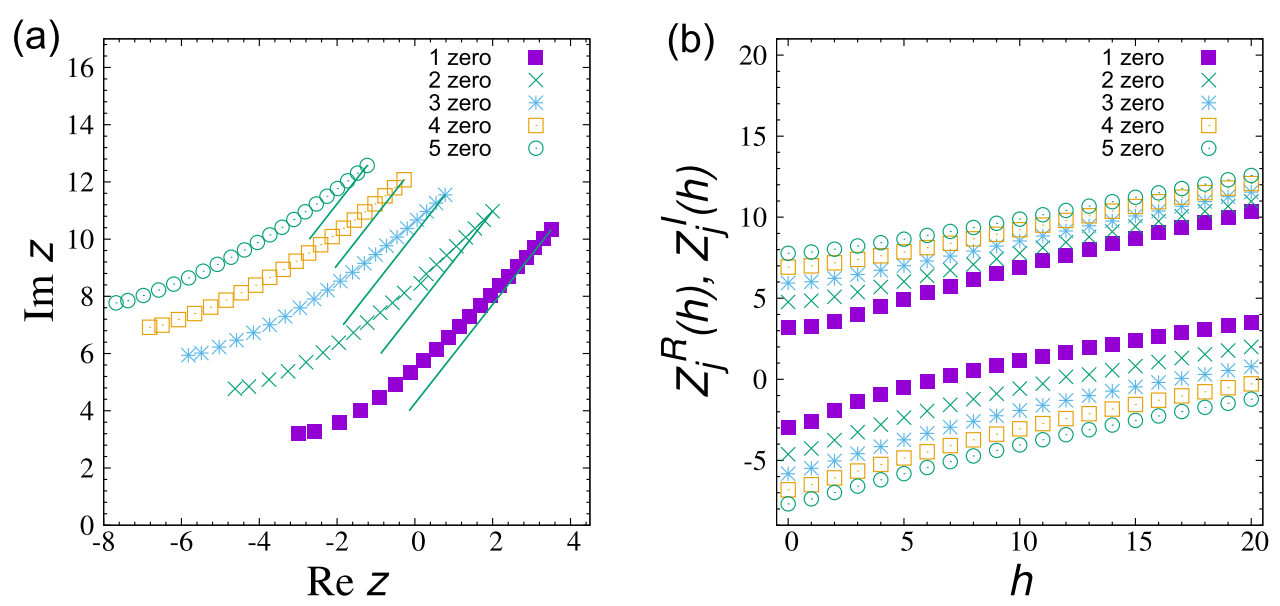

Figure 10. (a) Coordinates of the first five Fisher zeros of the partition function (39) in the complex $z$ plane for different values of the magnetic field $h_{j}=j, j=0,1, \ldots, 20$. The plots have a tendency to settle along lines forming an angle $\psi=\pi / 3$ with a real $z$ axis. (b) Scaling functions for the first five zeros $\mathcal{Z}_{j}^{\mathcal{R}}(h)$ (lower plots), $\mathcal{Z}_{j}^{\mathcal{I}}(h)$ (upper plots) for the real and imaginary part of the first Fisher zeros coordinate, equation (47), as functions of the scaling variable $h=H N^{1+\frac{\beta}{\alpha-2}}$.

\subsection{Motion of the Fisher zeros in a real external field}

In the final part of this section we analyse the motion of the Fisher zeros in the presence of a (real) external field [5]. Since the Lee-Yang zeros of the model under consideration have purely imaginary coordinates, in the vicinity of the critical point the lines of motion of Fisher zeros form an angle $\psi$ that encodes order parameter critical exponents via equation (12). In our case, for the values of the exponents $\beta$ and $\delta$ given by (17) one gets for the angle:

$$
\psi=\frac{\pi}{3} \text {. }
$$

The expression for the approximated partition function in the vicinity of the critical point is obtained from (19) and reads

$$
Z_{N}^{\exp }(t, H)=\int_{-\infty}^{+\infty} \exp \left(\frac{-t x^{2}}{2}-\frac{x^{4}}{12 N}+x \sqrt{N} H\right) \mathrm{d} x
$$

To get (38) we have kept, as before, only the leading order contributions in $H$ and $t$ terms. As was pointed above, in this case the approximated expressions for the partition function attain the same form both in $t$ - and $\tau$ - representations. Therefore, the analysis of the Fisher zeros presented below concerns both the complex $t$ - and complex $\tau$-planes equally well. The expansion (38) can be conveniently rewritten in the rescaled variables $z, h$, equations (25), (35):

$$
Z^{\exp }(z, h)=\int_{0}^{\infty} \mathrm{e}^{-z x^{2}-x^{4}+h x} \mathrm{~d} x
$$

Note that for $h=0$ or $z=0$ (39) reduces to equations (24) or (34) respectively.

Figure 10(a) shows the coordinates of the first five Fisher zeros for different values of the (real) magnetic field in the complex $z$ plane. The coordinates have been calculated for different values of the magnetic field $h_{j}=j, j=0,1, \ldots, 20$. For $h_{j}=0$ we recover the values of 
the coordinates shown in figure 5. One can see the tendency of the zeros to settle along a line forming an angle $\psi=\frac{\pi}{3}$ with the real $z$ axis (shown by a solid line in the figure). However, the asymptotics are preceded by a crossover region for very small values of $h$. Similar tendency for a first few zeros of a 3D Ising model was observed in [5] and explained by the finite-size corrections [28]. The closer the zeros to the critical point, the smaller the crossover region.

To further analyse motion of the zeros we apply the FSS as outlined below. According to the FSS theory [29], for a $d$-dimensional system in the vicinity of the critical point one expects the following scaling for the real and imaginary parts of the $j$ th zero coordinate in the reduced temperature $t$ plane:

$$
\begin{aligned}
& \operatorname{Re} t_{j}(N, H)=b^{-1 / \nu} \mathcal{T}_{j}^{\mathcal{R}}\left(H b^{y^{H}}, N b^{-d}\right), \\
& \operatorname{Im} t_{j}(N, H)=b^{-1 / \nu} \mathcal{T}_{j}^{\mathcal{I}}\left(H b^{y^{H}}, N b^{-d}\right) .
\end{aligned}
$$

Here, $b$ is the scaling factor, $\nu$ and $y_{H}$ are the correlation length critical exponent and field scaling dimension, $\mathcal{T}_{j}^{\mathcal{R}}(x, y)$ and $\mathcal{T}_{j}^{\mathcal{I}}(x, y)$ are the scaling functions. Being generalized homogeneous functions of two variables, the scaling functions can be rewritten as functions of a single conveniently chosen scaling variable. Choosing the factor $b=N^{-1 / d}$ the expressions (40), (41) attain the following form:

$$
\begin{aligned}
& \operatorname{Re} t_{j}(N, H)=N^{-1 / d \nu} \mathcal{T}_{j}^{\mathcal{R}}\left(H N^{-y_{H} / d}, 1\right), \\
& \operatorname{Im} t_{j}(N, H)=N^{-1 / d \nu} \mathcal{T}_{j}^{\mathcal{I}}\left(H N^{-y_{H} / d}, 1\right) .
\end{aligned}
$$

Making use of the familiar (hyper)scaling relations $\left(d \nu=2-\alpha, y_{h} / d=1-\beta / 2-\alpha\right)$ we get the following expressions for the motion of zeros:

$$
\begin{aligned}
& \operatorname{Re} t_{j}(N, H)=N^{\frac{1}{\alpha-2}} \mathcal{T}_{j}^{\mathcal{R}}\left(H N^{1+\frac{\beta}{\alpha-2}}\right), \\
& \operatorname{Im} t_{j}(N, H)=N^{\frac{1}{\alpha-2}} \mathcal{T}_{j}^{\mathcal{I}}\left(H N^{1+\frac{\beta}{\alpha-2}}\right),
\end{aligned}
$$

where we have introduced the single variable functions:

$$
\mathcal{T}_{j}^{\mathcal{R}}(x) \equiv \mathcal{T}_{j}^{\mathcal{R}}(x, 1), \mathcal{T}_{j}^{\mathcal{I}}(x) \equiv \mathcal{T}_{j}^{\mathcal{I}}(x, 1) .
$$

Note that relations (44), (45) contain the system size only via the number of particles $N$ (and not via the spacial extent and dimension) and therefore are convenient for using them for systems on graphs, where the notion of Euclidean dimension is not defined. Equations (44), (45) can be rewritten in the rescaled variables $z, h$ (see equations (25) and (35)):

$$
\begin{aligned}
& \operatorname{Re} z_{j}=\mathcal{Z}_{j}^{\mathcal{R}}(h), \\
& \operatorname{Im} z_{j}=\mathcal{Z}_{j}^{\mathcal{I}}(h),
\end{aligned}
$$

where

$$
\mathcal{Z}_{j}^{\mathcal{R}}(h) \equiv \sqrt{3} \mathcal{T}_{j}^{\mathcal{R}}(h / \sqrt[4]{12}), \quad \mathcal{Z}_{j}^{\mathcal{I}}(h) \equiv \sqrt{3} \mathcal{T}_{j}^{\mathcal{I}}(h / \sqrt[4]{12}) .
$$

We plot the scaling functions (48) in figure 10(b) for the first five zeros $j=1, \ldots, 5$. The prominent feature of the plot is that a ratio of the values of the scaling functions at $h=0$ gives the value of the Fisher pinching angle:

$$
\mathcal{T}_{j}^{\mathcal{I}}(0) / \mathcal{T}_{j}^{\mathcal{R}}(0)=\mathcal{Z}_{j}^{\mathcal{I}}(0) / \mathcal{Z}_{j}^{\mathcal{R}}(0)=\tan \varphi
$$


Indeed, with the value $\tan \varphi=\tan \pi / 4=-1$, equation (22), one gets $\mathcal{T}_{j}^{\mathcal{I}}(0)=-\mathcal{T}_{j}^{\mathcal{R}}(0)$ or $\mathcal{Z}_{j}^{\mathcal{I}}(0)=-\mathcal{Z}_{j}^{\mathcal{R}}(0)$ as is nicely observed in the figure.

\section{Ising model on an annealed scale-free network}

Now, with knowledge of the behaviour of the partition function zeros on a complete graph to hand, we consider the critical behaviour of the Ising model on complex networks, i.e. on a random graph [12]. The Hamiltonian of the model in this case reads

$$
-\mathcal{H}=\frac{1}{2} \sum_{l \neq m} J_{l m} S_{l} S_{m}+H \sum_{l} S_{l} .
$$

Here, the sums are performed over all graph nodes $l, m$ and the adjacency matrix $J$ stores all information about the graph structure: the matrix elements $J_{l m}=1$ if the nodes are linked and $J_{l m}=0$ otherwise. In random graphs different nodes have different number of links (different node degrees $K$ ). The node degrees are random variables and inherent features of their distribution $p(K)$ appear to play one of the key roles governing universal critical behaviour [13]. As a first instance of a complex network we will consider an annealed network. This network is defined as an ensemble of all networks consisting of $N$ nodes with a given degree sequence $\left\{K_{1}, K_{2}, \ldots, K_{N}\right\}$, maximally random under the constraint that their degree distribution is a given one, $p(K)$ (see e.g. [14, 17]). Such a construction resembles the uncorrelated configuration model of a complex network (see e.g. [30]). However the latter is an example of a quenched network, whereas for the Ising model on an annealed network, the graph configuration is also fluctuating just like Ising spins do. When thermodynamic properties are calculated, the presence of quenched disorder is taken into account by averaging the free energy over different disorder configurations, whereas in the annealed case the partition function is averaged [15]. Therefore, considering the Ising model on an annealed network, we will be interested in the behaviour of the partition function averaged with respect to different network configurations.

\subsection{Partition function}

For the Hamiltonian (50) the corresponding partition function is obtained by:

$$
Z_{N}(T, H)=\operatorname{Tr}_{S} \operatorname{Tr}_{J} \mathrm{e}^{-\beta \mathcal{H}}
$$

As in (14), the first trace is taken over the spin system: $\operatorname{Tr}_{S}(\ldots)=\prod_{l} \sum_{S_{l}= \pm 1}(\ldots)$, whereas the second one means an averaging with respect to the distribution of the network links $\mathcal{P}(J): \operatorname{Tr}_{J}(\ldots)=\prod_{l, m, l \neq m} \sum_{J_{l m}=0,1} \mathcal{P}(J)(\ldots)$.

To construct an annealed network of $N$ nodes $l=1, \ldots, N$, each node $l$ is assigned a label $k_{l}$ and the probability of a link between nodes $l$ and $m$ is defined as:

$$
p_{l m}=\frac{k_{l} k_{m}}{N\langle k\rangle},
$$

where $\langle k\rangle=\frac{1}{N} \sum_{l} k_{l}$. The variables $k$ are taken from the distribution $p(k)$ and indicate the expected node degree. Indeed, it is straightforward to show that the expected value of the node degree $\mathbb{E} K_{l}=\sum_{m} p_{l m}=k_{l}$. One can show [14] that averaging (51) over the distribution of network links with probability function (52) leads to the following expression for the partition function: 


$$
Z_{N}(T, H)=\operatorname{Tr}_{S} \exp \left(\frac{1}{2 N\langle k\rangle T} \sum_{l \neq m} S_{l} S_{m} k_{l} k_{m}+\frac{H}{T} \sum_{l} S_{l}\right) .
$$

An analogous expression for the partition function is usually obtained also within the mean field approximation for the Ising model on an uncorrelated quenched network [13, 30] (configuration model). For the annealed network however the factor in front of the double sum in (53) is a certain function of temperature [14]. Since in our study we are interested in the angles of incidence of partition function zeros, which are independent of any analytic parametrization of the temperature plane, we keep in (53) only a linear term of this function.

Since for the annealed network the interaction term in (50) attains a separable form, one can apply the Stratonovich-Hubbard transformation to (53) to take the trace exactly and to get the following expression for the partition function:

$$
\left.Z_{N}(T, H)=\int_{-\infty}^{+\infty} \exp \left(\frac{-N\langle k\rangle x^{2}}{2 T}+\sum_{l} \ln \cosh \left[\left(x k_{l}+H\right) / T\right)\right]\right) \mathrm{d} x
$$

As in the previous section, the prefactors are omitted here and below. Now, the sum over $l$ in the exponent (54) can be rewritten in terms of the integral over $k$ for a given distribution function $p(k)$ :

$$
\sum_{l} f\left(k_{l}\right)=N \int_{k_{\min }}^{k_{\max }} p(k) f(k) \mathrm{d} k,
$$

where $k_{\min }$ and $k_{\max }$ are the minimal and maximal values of the variable $k$. For further analysis it will be convenient to keep the integral in $x$ in the positive half-plane leading to the representation for the partition function

$$
\begin{aligned}
Z_{N}(T, H)= & \int_{0}^{+\infty} \mathrm{e}^{\frac{-\langle k\rangle x^{2} T}{2}}\left\{\exp \left[N \int_{k_{\min }}^{k_{\max }} \mathrm{d} k p(k) \ln \cosh \left(\frac{x k}{\sqrt{N}}+\frac{H}{T}\right)\right]\right. \\
& \left.+\exp \left[N \int_{k_{\min }}^{k_{\max }} \mathrm{d} k p(k) \ln \cosh \left(-\frac{x k}{\sqrt{N}}+\frac{H}{T}\right)\right]\right\} \mathrm{d} x
\end{aligned}
$$

For the complete graph of $N$ nodes substituting in (56) $p(k)=\delta(k-\mathrm{N}+1)$ one recovers (18). In this section we are interested in the scale-free networks, when the function $p(k)$ is given by a power law

$$
p(k)=c_{\lambda} k^{-\lambda},
$$

with a normalizing constant $c_{\lambda}$. Note, that scale-free networks with $k_{\min }=1$ do not possess a spanning cluster for $\lambda>\lambda_{\mathrm{c}}\left(\lambda_{\mathrm{c}}=4\right.$ for continuous degree distribution and $\lambda_{\mathrm{c}} \simeq 3.48$ for the discrete one [31]). To avoid this restriction, without loss of generality we choose from now on $k_{\min }=2$, whereas for the upper integration boundary in (55) we take in the thermodynamic $\operatorname{limit}^{6} \lim _{N \rightarrow \infty} k_{\max } \rightarrow \infty$. Then, for the scale-free network, equation (56) can be conveniently rewritten as:

$$
Z_{N}(T, H)=\int_{0}^{+\infty} \mathrm{e}^{\frac{-\langle k\rangle x^{2} T}{2}}\left\{\exp \left[I_{\lambda}^{+}(x)\right]+\exp \left[I_{\lambda}^{-}(x)\right]\right\} \mathrm{d} x
$$

\footnotetext{
6 The leading in $N$ value of this integral representation does not depend on the way in which $k_{\max }$ tends to infinity. These are the next-leading terms that will depend on the $N$ dependency of the upper cut-off. See appendix A for more details.
} 
where

$$
I_{\lambda}^{ \pm}(x)=c_{\lambda}\left(\frac{x}{\sqrt{N}}\right)^{\lambda-1} N \int_{\frac{2 x}{\sqrt{N}}}^{\infty} \frac{1}{y^{\lambda}} \ln \cosh \left( \pm y+\frac{H}{T}\right) \mathrm{d} y
$$

Consider the integral in (59). For large $y$ the integrand decays as $y^{1-\lambda}$ and the integral is finite at the upper integration boundary for the values $\lambda>3$ we are interested in. However, for small $y$ the integrand behaves as $( \pm y+H / T)^{2} y^{-\lambda}+\ldots$ and leads to divergent terms when the lower integration boundary is set to zero, i.e. in the thermodynamic limit $N \rightarrow \infty$. These divergent terms do not appear in the whole expression since they are canceled by the $N$ dependent prefactor in (59). To single them out and to show this cancelation explicitly, it is instructive to consider the function $I_{\lambda}^{ \pm}(x)$ (59) for different values of $\lambda$ (see e.g. [32] for a more detailed account). As in the former section, we make asymptotic estimates for small $H$. Leading terms of the resulting expressions read:

$$
\begin{aligned}
& I_{\lambda}^{ \pm}(x)=N\left[\frac{\left\langle k^{2}\right\rangle}{2} \frac{x^{2}}{N}-a(\lambda)\left(\frac{x}{\sqrt{N}}\right)^{\lambda-1} \pm \frac{\langle k\rangle H x}{T \sqrt{N}}\right], \quad 3<\lambda<5, \\
& I_{\lambda}^{ \pm}(x)=N\left[\frac{\left\langle k^{2}\right\rangle}{2} \frac{x^{2}}{N}-\frac{\left\langle k^{4}\right\rangle}{12}\left(\frac{x}{\sqrt{N}}\right)^{4} \pm \frac{\langle k\rangle H x}{T \sqrt{N}}\right], \quad \lambda>5,
\end{aligned}
$$

where the numerical of the coefficients $a(\lambda)=-c_{\lambda} \int_{0}^{\infty} \mathrm{d} y y^{-\lambda}\left(\ln \cosh y-y^{2} / 2\right), a(\lambda)>0$, are listed for different $\lambda$ in [32] and the moments of the variable $k$ are calculated with the distribution (57). The case $\lambda=5$ is to be considered separately. Integrating out the logarithmic singularity one gets for the first leading terms:

$$
I_{\lambda}^{ \pm}(x)=N\left[\frac{\left\langle k^{2}\right\rangle}{2} \frac{x^{2}}{N}-\left(\frac{x}{\sqrt{N}}\right)^{4} \frac{\ln N}{24} \pm \frac{\langle k\rangle H x}{T \sqrt{N}}\right] .
$$

With the expressions (60)-(62) for $I_{\lambda}^{ \pm}(x)$ we are in a position to analyse the partition function (58) at different values of $\lambda$. As is well established by now, critical behaviour of a system on a scale-free network with the partition function (58) depends of the value of the exponent $\lambda$ in an essential way $[12,13]$. In particular, the system remains ordered for any finite temperature at $\lambda \leqslant 3$. A second order phase transition at finite temperature occurs for $\lambda>3$. It is governed by standard mean field exponents (17) in the region $\lambda \geqslant 5$ with logarithmic corrections at $\lambda=5$, however the exponents attain $\lambda$-dependency for $3<\lambda<5$ [30]:

$$
\alpha=(\lambda-5) /(\lambda-3), \beta=1 /(\lambda-3), \delta=\lambda-2, \gamma=1
$$

The mean-field approximation delivers the classical value for the magnetic susceptibility exponent $\gamma=1$ for any $\lambda>3$. The other exponents however become $\lambda$ dependent at $3<\lambda<5$. This is because the Ising model on complex scale-free networks follows a heterogeneous rather than homogeneous mean-field.

Our task will be to describe the phase transition in the Ising model on an annealed scalefree network in terms of the partition function zeros, similarly as it was done for the complete graph in the previous section. Substituting values of the exponents (63) together with the critical amplitude ratio $A_{+} / A_{-}=0$ [33] into relations (3), (32) we get for the region $3<\lambda<5$ : 


$$
\begin{aligned}
& \varphi=\frac{\pi(\lambda-3)}{2(\lambda-1)}, \\
& \sigma=\frac{\lambda-2}{\lambda-1} .
\end{aligned}
$$

Let us check if these values can be obtained directly from the analysis of the zeros of the partition function (58) in complex $T$ and $H$ planes.

\subsection{Fisher zeros for the Ising model on an annealed scale-free network at $H=0$}

Substituting expressions for $I \pm_{\lambda}(x)(60)-(62)$ at $H=0$ into the partition function (58) at different values of $\lambda$ we get:

$$
Z_{N}(t)=\left\{\begin{array}{lcc}
\int_{0}^{+\infty} \exp \left[-\frac{\left\langle k^{2}\right\rangle x^{2} t}{2}-a(\lambda) N\left(\frac{x}{\sqrt{N}}\right)^{\lambda-1}\right] \mathrm{d} x, 3<\lambda<5, & \lambda=5, \\
\int_{0}^{+\infty} & \exp \left[-\frac{\left\langle k^{2}\right\rangle x^{2} t}{2}-\frac{x^{4}}{N} \frac{\ln N}{24}\right] \mathrm{d} x, & \lambda=5, \\
\int_{0}^{+\infty} & \exp \left[-\frac{\left\langle k^{2}\right\rangle x^{2} t}{2}-\frac{\left\langle k^{4}\right\rangle}{12} \frac{x^{4}}{N}\right] \mathrm{d} x, & \lambda>5
\end{array}\right.
$$

with $t=\left(T-T_{\mathrm{c}}\right) / T_{\mathrm{c}}$ and the (pseudo)critical temperature $T_{\mathrm{c}}=\left\langle k^{2}\right\rangle /\langle k\rangle{ }^{7}$ Similar to the previous section, the partition function can be conveniently represented in terms of a single variable $z$ that combines $t$ and $N$ dependencies, see equation (25). However, now this variable differs in different regions of $\lambda$ :

$$
z=\left\{\begin{array}{cc}
t \frac{\left\langle k^{2}\right\rangle}{2}[a(\lambda)]^{2 /(\lambda-1)} N^{\frac{\lambda-3}{\lambda-1}}, & 3<\lambda<5, \\
t\left\langle k^{2}\right\rangle \sqrt{6 N} / \sqrt{\ln N}, & \lambda=5, \\
t\left\langle k^{2}\right\rangle \sqrt{3 N\left\langle k^{4}\right\rangle}, & \lambda>5 .
\end{array}\right.
$$

Written in terms of the variables appearing in (67), the partition function (66) has a simple form:

$$
Z(z)=\left\{\begin{array}{l}
\int_{0}^{+\infty} \exp \left(-z x^{2}-x^{\lambda-1}\right) \mathrm{d} x, \quad 3<\lambda<5, \\
\int_{0}^{+\infty} \exp \left(-z x^{2}-x^{4}\right) \mathrm{d} x, \quad \lambda \geqslant 5 .
\end{array}\right.
$$

Two obvious conclusions follow: (i) since the functional form of $Z(z)$ dependency at $\lambda=5$ and $\lambda>5$ is the same, the location of the Fisher zeros in the complex $z$ plane will be the same too. This leads to the conclusion that an impact angle $\varphi$ that corresponds to the Ising model on an annealed network does not change for $\lambda \geqslant 5$ and (ii) these expressions coincide with the partition function of the Ising model on the complete graph, equation (24). Therefore, analysis of the Fisher zeros of the last model, performed in section 3.1 applies equally well to the Fisher zeros of the Ising model on an annealed network. In particular, one concludes, that:

$$
\varphi=\pi / 4, \quad \lambda \geqslant 5 \text {. }
$$

${ }^{7}$ For a finite-size system, $T_{\mathrm{c}}$ depends on $N$ via the $N$-dependency of the moments $\langle k\rangle,\left\langle k^{2}\right\rangle$. 


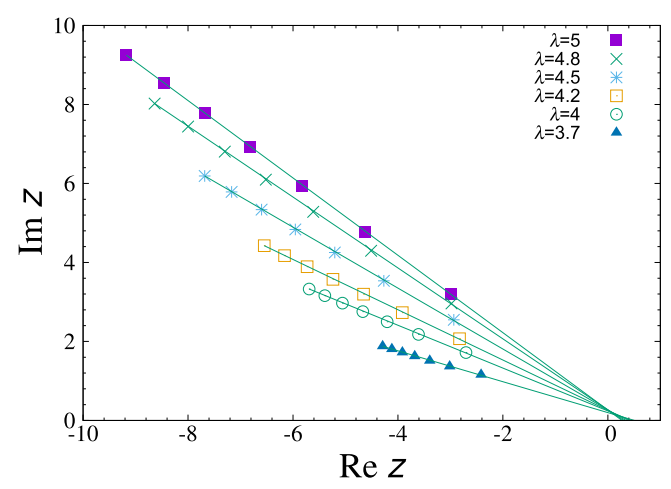

Figure 11. Fisher zeros for the Ising model on an annealed scale-free network at zero magnetic field for different $\lambda$. The zeros have a clear tendency to situate along the straight lines crossing the real $z$ axis in the vicinity of the critical point $z_{\mathrm{c}}$. The angles formed by each of the lines with the real $z$ axis decrease in the region $3<\lambda<5$ as predicted by equation (64).

Table 2. Numerically calculated values of the angle $\varphi$ for different $\lambda$. The angle is calculated by linear fitting of Fisher zeros with the indices $j=j_{\min }, \ldots, 7$. The confidence interval, when not written explicitly, is less than the last significant digit. The last row gives $\varphi$ predicted by the analytic formula (64).

\begin{tabular}{lcccccc}
\hline$j_{\min }$ & $\lambda \geqslant 5$ & $\lambda=4.8$ & $\lambda=4.5$ & $\lambda=4.2$ & $\lambda=4$ & $\lambda=3.7$ \\
\hline 1 & $0.246(5) \pi$ & $0.233(1) \pi$ & $0.209(1) \pi$ & $0.180(1) \pi$ & $0.158(1) \pi$ & $0.117(2) \pi$ \\
2 & $0.248 \pi$ & $0.234 \pi$ & $0.210 \pi$ & $0.182 \pi$ & $0.160(1) \pi$ & $0.121(1) \pi$ \\
3 & $0.248 \pi$ & $0.234 \pi$ & $0.211 \pi$ & $0.183 \pi$ & $0.162 \pi$ & $0.123 \pi$ \\
4 & $0.248 \pi$ & $0.235 \pi$ & $0.212 \pi$ & $0.184 \pi$ & $0.162 \pi$ & $0.124 \pi$ \\
5 & $0.248 \pi$ & $0.235 \pi$ & $0.212 \pi$ & $0.184 \pi$ & $0.163 \pi$ & $0.125 \pi$ \\
6 & $0.249 \pi$ & $0.235 \pi$ & $0.212 \pi$ & $0.185 \pi$ & $0.163 \pi$ & $0.125 \pi$ \\
Exact & $0.250 \pi$ & $0.237 \pi$ & $0.214 \pi$ & $0.188 \pi$ & $0.167 \pi$ & $0.130 \pi$ \\
\hline
\end{tabular}

Let us note as well, that the logarithmic corrections to scaling appear in the marginal case $\lambda=5$ [33-36]. Such a logarithmic correction appears in $I_{\lambda}^{ \pm}(x)$ at $\lambda=5$ too. However, it does not contribute to the terms leading in $1 / N$, resulting in the conclusion, that the impact angle of Fisher zeros (and, therefore, the leading exponent for the heat capacity) is the same at $\lambda=5$ and $\lambda>5$.

Similar to the preceding sections for the Ising model on the complete graph, to proceed with the analysis of the Fisher zeros at $3<\lambda<5$, we calculate the numerical values of the coordinates of several first zeros as shown in figure 11. As is seen from the figure, the zeros have a clear tendency to situate along the straight lines crossing the real $z$ axis in the vicinity of the critical point $z_{\mathrm{c}}$. Moreover, the angle formed by each of the lines differs for different $\lambda$ and decreases with $\lambda$. To study this dependency in more detail, for each value of $\lambda$ we fit $j$ Fisher zeros in the interval $j=j_{\min }, \ldots, j_{\max }$ for $j_{\max }=7$ and give the resulting estimate for $\varphi$ in table 2 . One can see that the values determined numerically approach those predicted by an analytic formula (64), the higher the order of the zeros used for the fit, the higher the accuracy. This tendency is quite similar to those observed for the Fisher zeros on a complete graph (see figure 6). To further demonstrate this resemblance, we plot in figure 12(a) the 

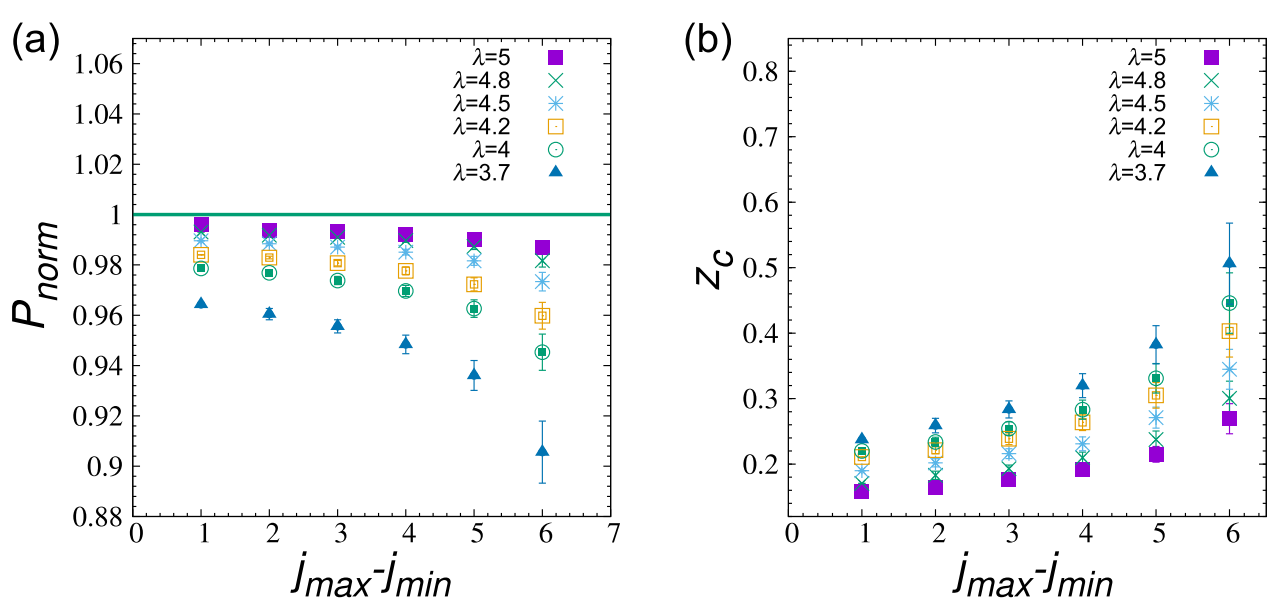

Figure 12. Values (a) of the ratio $P_{\text {norm }}=\varphi / \frac{\pi(\lambda-3)}{2(\lambda-1)}$ and (b) of estimates for the critical temperature $z_{\mathrm{c}}$ for the partition function (68) obtained by fitting of $j$ Fisher zeros in the interval $j=j_{\min }, \ldots, j_{\max }$ for $j_{\max }=7$ and different values of $j_{\min }$. A solid line in panel (a) shows an exact value $P_{\text {norm }}=1$.

Table 3. Linear fitting of the function $\ln \left|z_{j}\right|=a+b \ln j$ for the Fisher zeros with indices $j=j_{\min }, \ldots, 7$ at different $\lambda$. The table shows results for the angle coefficient $b$. The confidence interval, when not written explicitly, is less than the last significant digit. The last row gives the value $b=1 /(2-\alpha)$ as predicted by an analytic formula (71).

\begin{tabular}{lcccccc}
\hline$j_{\text {min }}$ & $\lambda>5$ & $\lambda=4.8$ & $\lambda=4.5$ & $\lambda=4.2$ & $\lambda=4$ & $\lambda=3.7$ \\
\hline 1 & $0.558(8)$ & $0.528(7)$ & $0.477(6)$ & $0.417(5)$ & $0.370(5)$ & $0.286(3)$ \\
2 & $0.537(4)$ & $0.509(3)$ & $0.460(3)$ & $0.402(3)$ & $0.357(2)$ & $0.277(2)$ \\
3 & $0.529(2)$ & $0.501(2)$ & $0.453(2)$ & $0.396(2)$ & $0.352(1)$ & $0.274(1)$ \\
4 & $0.525(1)$ & $0.497(1)$ & $0.450(1)$ & $0.393(1)$ & $0.349(1)$ & $0.271(1)$ \\
5 & $0.522(1)$ & $0.494(1)$ & $0.447(1)$ & $0.391(1)$ & $0.348(1)$ & $0.270(1)$ \\
6 & 0.520 & 0.492 & 0.445 & 0.390 & 0.346 & 0.269 \\
Exact & 0.500 & 0.474 & 0.429 & 0.375 & 0.333 & 0.259 \\
\hline
\end{tabular}

dependency of the ratio of the numerically calculated angle $\varphi$ to its value predicted by formula (64): $P_{\text {norm }}=\varphi / \frac{\pi(\lambda-3)}{2(\lambda-1)}$. This ratio tends to $P_{\text {norm }}=1$ with an increase of the order of the zeros used for the fit. One can notice a similar tendency for the behaviour of the critical temperature $z_{\mathrm{c}}$, see figure 12(b). Again one observes similarity with the behaviour of $z_{\mathrm{c}}$ for the Ising model on a complete graph, see figure 6(b).

Another inherent feature of the locations of the Fisher zeros is that the distance between two successive zeros decreases with increasing index (see figure 11 for the annealed scale-free network as well as figure 5 for the complete graph). Indeed, taken that the FSS of the $j$ th zero $t_{j}$ in the complex $t$ plane for large $j$ is given by [5]:

$$
t_{j} \sim\left(\frac{j}{N}\right)^{\frac{1}{2-\alpha}}
$$


one arrives at the conclusion that $\Delta t_{j} \equiv t_{j}-t_{j-1} \sim j^{\kappa}$, with $\kappa=(\alpha-1) /(2-\alpha)$. The last exponent is negative for the values of $\alpha$ we are interested in: $\kappa=-1 / 2, \lambda \geqslant 5$ and $\kappa=-2$ / $(\lambda-1), 3<\lambda<5$. Rewriting (70) in terms of $z_{j}$ (67) gives:

$$
z_{j} \sim j^{\frac{1}{2-\alpha}}
$$

To check how does the scaling of the Fisher zeros hold with $j$, we calculate the value of the exponent in (71) fitting the function $\ln \left|z_{j}\right|=a+b \ln j$ for the Fisher zeros with indices $j=j_{\min }, \ldots, 7$ at different $\lambda$. The results are compared with the exact value in table 3 . One can see the right tendency of approach of the numerically calculated numbers to their exact counterparts with an increase of $j$.

In order to check scaling of the Fisher zeros $t_{j}$ with $N$, there is no need to calculate explicitly their coordinates at different values of $N$. Indeed, the $t_{j}(N)$ dependency follows from the $z(t, N)$ functional form, as given by (67). Expressing $t$ from there one gets that the power law scaling $t_{j} \sim N^{1 /(\alpha-2)}$ holds for $3<\lambda<5$ and $\lambda>5$ whereas it is enhanced by a logarithmic correction at $\lambda=5: t_{j} \sim N^{-1 / 2}(\ln N)^{1 / 2}$.

\subsection{Lee-Yang zeros for an annealed scale-free network at $T=T_{c}$ and motion of Fisher zeros in the magnetic field}

Substituting expressions for $I_{\lambda}^{ \pm}(x)(60)-(62)$ at $T=T_{\mathrm{c}}=\left\langle k^{2}\right\rangle /\langle k\rangle$ into the partition function (58) at different values of $\lambda$ and keeping the leading contributions in $1 / N$ we get:

$$
Z(h)=\left\{\begin{array}{l}
\int_{0}^{+\infty} \exp \left(-x^{\lambda-1}\right) \cosh (h x) \mathrm{d} x, \quad 3<\lambda<5, \\
\int_{0}^{+\infty} \exp \left(-x^{4}\right) \cosh (h x) \mathrm{d} x, \quad \lambda \geqslant 5 .
\end{array}\right.
$$

Here, the $H$ - and $N$-dependencies of the partition function are adsorbed in a single variable $h$. Its explicit form differs for different regions of $\lambda$ :

$$
h=\left\{\begin{array}{lc}
H \frac{\langle k\rangle^{2}}{\left\langle k^{2}\right\rangle} a(\lambda)^{1 /(1-\lambda)} N^{\frac{\lambda-2}{\lambda-1}}, & 3<\lambda<5, \\
H \frac{\langle k\rangle^{2}}{\left\langle k^{2}\right\rangle}\left(\frac{24}{\ln N}\right)^{1 / 4} N^{3 / 4}, & \lambda=5, \\
H \frac{\langle k\rangle^{2}}{\left\langle k^{2}\right\rangle}\left(\frac{12}{\left\langle k^{4}\right\rangle}\right)^{1 / 4} N^{3 / 4}, & \lambda>5 .
\end{array}\right.
$$

Similar to the discussion for the $t_{j}(N)$ scaling at the end of the previous subsection, here, the scaling of $h_{j}(N)$ directly follows from the $h(H, N)$ dependency given by (73). Comparing (73) with the definition (31) one gets:

$$
\sigma=\left\{\begin{array}{lc}
\frac{\lambda-2}{\lambda-1}, & 3<\lambda<5 \\
3 / 4, & \lambda>5
\end{array}\right.
$$

The logarithmic correction appears at $\lambda=5: H_{j} \sim N^{-3 / 4}(\ln N)^{1 / 4}$.

In figure 13 we plot numerically obtained lines of zeros for the real and imaginary parts of the partition function (72) at $T=T_{\mathrm{c}}$ and different values of $\lambda$ in the complex magnetic field $h=\operatorname{Re} h+\mathrm{i} \operatorname{Im} h$ plane. The points where the lines of different colour cross give the coordinates of the Lee-Yang zeros. Note that whereas the coordinate of the first Lee-Yang zero (that closest to the origin) is purely imaginary for any value of $3<\lambda<5$ (and it is its presence that allows to make conclusions about the scaling with $N$ governed by the exponent (74)), it is not the case for the zeros of higher order: the number of zeros with $\operatorname{Re} h_{j}=0$ decreases with $j$. 

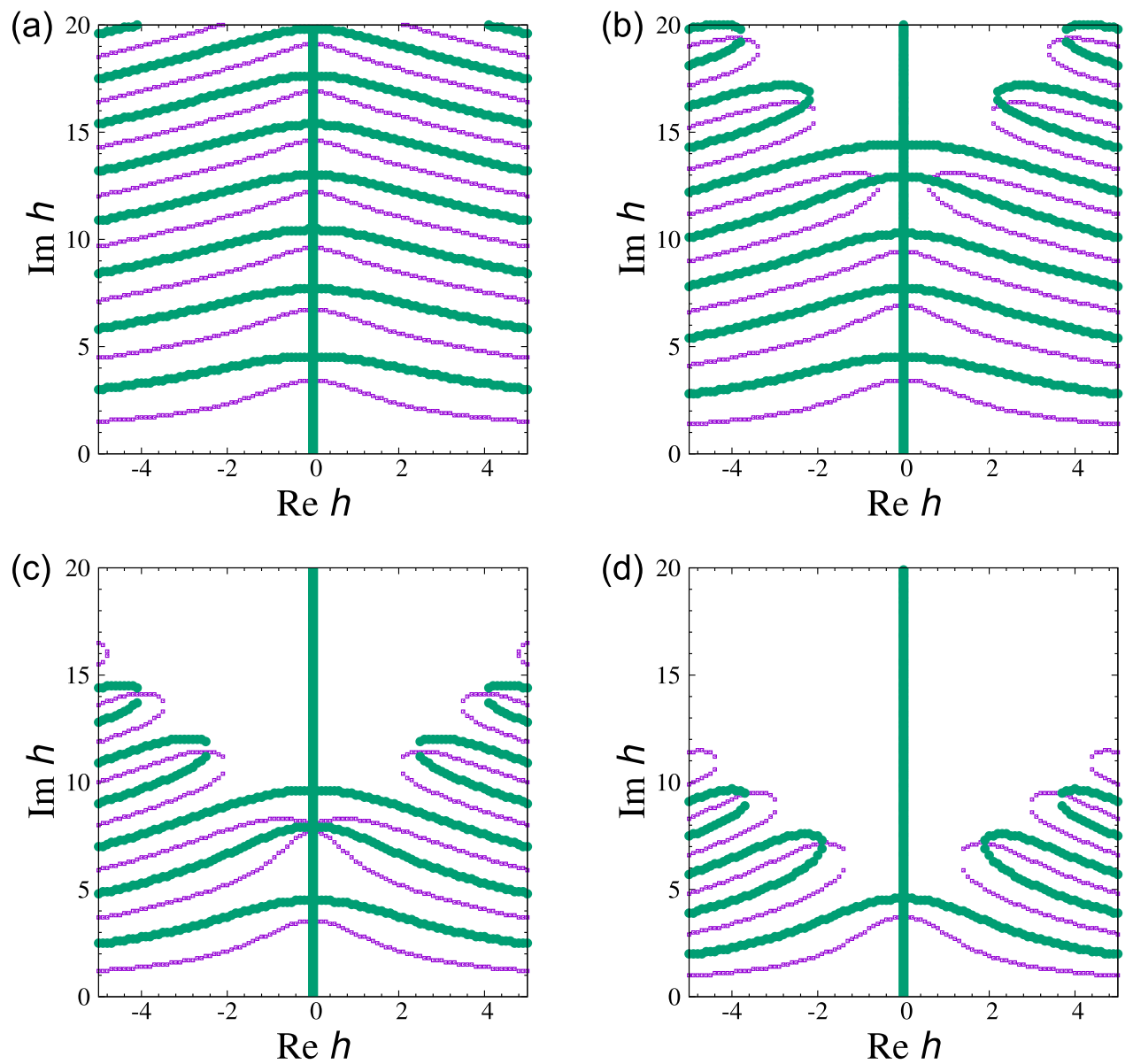

Figure 13. Lines of zeros for the real and imaginary part of the partition function (72) at $T=T_{\mathrm{c}}$ and different values of $\lambda$ in the complex magnetic field plane,thin and thick curves (violet and green dots online) respectively: (a) $\lambda \geqslant 5$; (b) $\lambda=4.5$; (c) $\lambda=4$; (d) $\lambda=3.5$. The points where the lines of different colours cross give the coordinates of the Lee-Yang zeros. Note that one of the $\operatorname{Im} Z=0$ lines coincides with the vertical axis in the plot.

In this respect the behaviour of Lee-Yang zeros for the Ising model on an annealed scale-free network differs crucially from the behaviour on the complete graph (see the previous section). Whereas in the complete graph the zeros were entirely imaginary and obeyed the Lee-Yang circle theorem, this theorem is violated in the case of the annealed scale-free network.

As seen in figure 13, the imaginary part of $Z(h)$ vanishes when $h$ itself is imaginary. This is because the partition function is an even function of $h$. The intersections of the different types of contour on the plot give the locations of the Lee-Yang zeros. When $\lambda \geqslant 5$ all zeros are on the imaginary axis. But for $\lambda=4.5$ only first three zeros are imaginary and zeros of higher order $\left(h_{j}\right.$ for $j>3$ ) have non-vanishing real parts. Similar behaviour is found for all values of $\lambda$ between 3 and 5; there is a number, $\mathcal{N}$, finite in size, such that $h_{j}$ is purely imaginary for $j \leqslant \mathcal{N}$ but has non-vanishing real part for $j>\mathcal{N}$. The number $\mathcal{N}$ decreases with decreasing $\lambda$. 
Table 4. Coordinates of the few first Lee-Yang zeros $h_{j}$ for the Ising model on an annealed scale-free network for different $\lambda$. In the region $3<\lambda<5$ the number of purely imaginary zeros decreases with a decrease of $\lambda$. However, the coordinate of the first Lee-Yang zero remains purely imaginary for any value of $\lambda$.

\begin{tabular}{lcccc}
\hline$j \backslash \lambda$ & $\lambda>5$ & $\lambda=4.5$ & $\lambda=4$ & $\lambda=3.5$ \\
\hline$j=1$ & $\mathrm{i} 3.453$ & $\mathrm{i} 3.495$ & $\mathrm{i} 3.569$ & $\mathrm{i} 3.762$ \\
$j=2$ & $\mathrm{i} 6.784$ & $\mathrm{i} 6.933$ & $\mathrm{i} 7.823$ & $1.875+\mathrm{i} 7.212$ \\
$j=3$ & $\mathrm{i} 9.636$ & $\mathrm{i} 9.474$ & $\mathrm{i} 8.149$ & $3.659+\mathrm{i} 9.496$ \\
$j=4$ & $\mathrm{i} 12.229$ & $0.589+\mathrm{i} 12.848$ & $2.418+\mathrm{i} 11.466$ & $5.138+\mathrm{i} 11.351$ \\
$j=5$ & $\mathrm{i} 14.650$ & $2.297+\mathrm{i} 16.346$ & $4.014+\mathrm{i} 14.174$ & $6.435+\mathrm{i} 12.983$ \\
$j=6$ & $\mathrm{i} 16.945$ & $3.761+\mathrm{i} 19.405$ & $5.446+\mathrm{i} 16.574$ & $7.608+\mathrm{i} 14.470$ \\
$j=7$ & $\mathrm{i} 19.140$ & $5.130+\mathrm{i} 22.229$ & $6.767+\mathrm{i} 18.776$ & $8.690+\mathrm{i} 15.850$ \\
$j=8$ & $\mathrm{i} 21.254$ & $6.427+\mathrm{i} 24.886$ & $8.005+\mathrm{i} 20.835$ & $9.702+\mathrm{i} 17.146$ \\
$j=9$ & $\mathrm{i} 23.301$ & $7.667+\mathrm{i} 27.414$ & $9.176+\mathrm{i} 22.783$ & $10.656+\mathrm{i} 18.375$ \\
$j=10$ & $\mathrm{i} 25.289$ & $8.859+\mathrm{i} 29.838$ & $10.293+\mathrm{i} 24.642$ & $11.563+\mathrm{i} 19.548$ \\
\hline
\end{tabular}

The values of Lee-Yang zeros determined numerically are listed in table 4 for different values of $3<\lambda<\lambda_{\mathrm{uc}}=5$, and for $\lambda \geqslant 5$. When $\lambda=4, \mathcal{N}=3$ while for $\lambda=3.5, \mathcal{N}=1$. In this respect the behaviour of the zeros differs for $\lambda \geqslant 5$ (where it is the same as for the partition function of the Ising model on a complete graph) and for $3<\lambda<5$. In appendix $\mathrm{B}$ we confirm this observation showing that the asymptotic behaviour of the integral (72) for non-integer $3<\lambda<5$ qualitatively differs from that at $\lambda \geqslant 5$.

Since it has been established for the Ising model on a regular lattice [1], the Lee-Yang theorem has been extended to a wider class of regular lattice Ising models. Besides the classical lattice discrete spin models [37], the theorem also holds for continuous spin systems [38]; for quantum systems such as an ideal pseudospin $-1 / 2$ Bose gas in an external field and arbitrary external potential [39]; for non-equilibrium systems [40], which narrate the collective phenomena and biophysics processes [41, 42]. There are many models for which the Lee-Yang theorem does not hold: Ising models with antiferromagnetic interactions [43, 44]; with degenerated spins [45, 46]; models with multi-spin interactions at sufficiently high temperature [47, 48]; and van der Waals gases [49, 50]. The theorem also fails for the model for which the first order phase transition is observed: a high- $q$ Potts model and the BlumeCapel model [51, 52]. Theorem is violated for the certain quantum many-body systems: Ising ferromagnet with arbitrary spin [53] and the quantum isotropic Ising chain in a transverse field [54]. For a more detailed review see [55].

Consequently, the Ising model on an annealed scale-free network at $3<\lambda<5$ belongs to the group of models where the Lee-Yang theorem is violated, whereas the model on the complete graph obeys the theorem. The difference in behaviour of the Lee-Yang zeros of the Ising model on a complete graph and on an annealed scale-free network manifests itself also when one checks the scaling of the zero coordinates with $j$. The coordinate of the zeros being complex, the scaling in principle can be observed with respect to the real or imaginary part of the coordinates or their combination. Typical results of our calculations are demonstrated in figure 14, where we plot coordinates of $\operatorname{Im} h_{j}$, Re $h_{j}$ and $\left|h_{j}\right|$ for the first ten Lee-Yang zeros of the Ising model on an annealed scale-free network at fixed value of $\lambda=4.5$. The solid lines correspond to the expected value $\sigma(\lambda=4.5) \simeq 0.714$, see (74). One can see that the curves for $\operatorname{Im} h_{j}$ and $\left|h_{j}\right|$ are very close to each other, since the value of $\operatorname{Re} h_{j}$ is relatively small. Similar behaviour is observed for the other values of $\lambda$. In particular, for high $j$ the $\operatorname{Im} h_{j}$ and $\left|h_{j}\right|$ functions are governed by the power law asymptotics with an exponent given by (74). The 


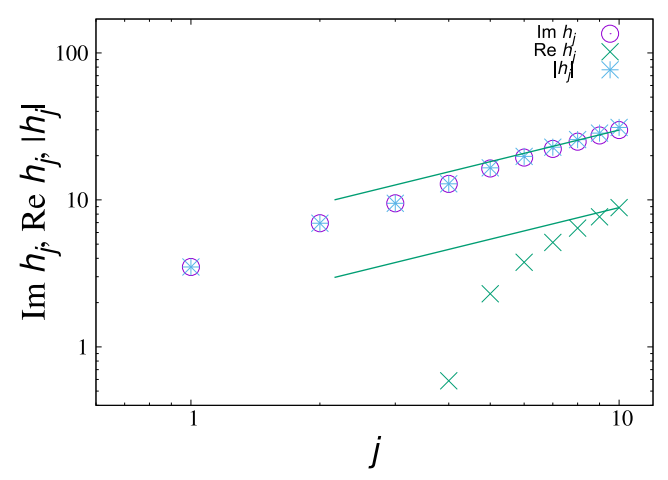

Figure 14. Coordinates of the first ten Lee-Yang zeros $h_{j}$ of the Ising model on an annealed scale-free network at $\lambda=4.5$. Different curves correspond to $\operatorname{Im} h_{j}, \operatorname{Re} h_{j},\left|h_{j}\right|$ as shown in the legend. One can see that the curves for $\operatorname{Im} h_{j}$ and $\left|h_{j}\right|$ are very close to each other (since the value of $\operatorname{Re} h_{j}$ is relatively small). The lines correspond to the expected value $\sigma(\lambda=4.5) \simeq 0.714$, from (74).
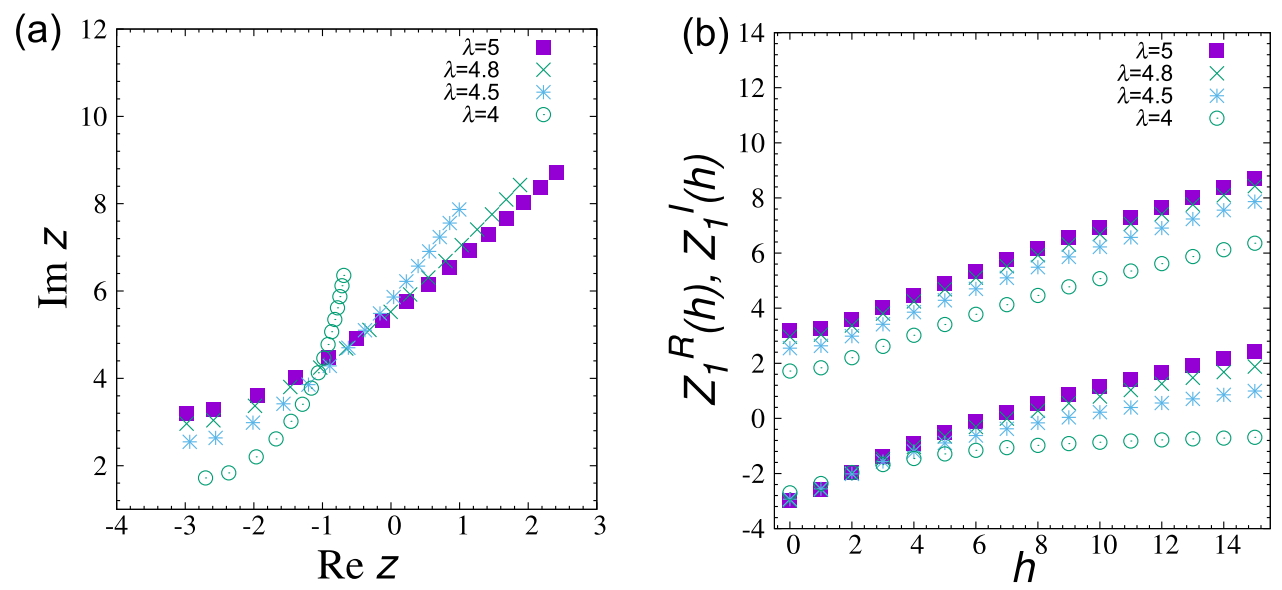

Figure 15. (a) Motion of the first Fisher zeros of the partition function (75) in the complex $z$ plane for different values of the real magnetic field $h=s, s=0,1, \ldots, 15$ and $\lambda$. (b) Scaling functions $\mathcal{Z}_{1}^{\mathcal{R}}(h)$ (lower plots), $\mathcal{Z}_{1}^{\mathcal{I}}(h)$ (upper plots) (77) for the real and imaginary part of the first Fisher zero coordinate $z_{1}$, as functions of the scaling variable $h$ at different values of $\lambda$.

power law behaviour is less pronounced for small $j$. Introducing a more general form of scaling that involves the parameter $C$ as it was done for the complete graph, see equation (31), does not help to improve the picture.

Let us next consider the motion of the Fisher zeros in the real magnetic field. To this end, similar as it was done in the former section for the Ising model on the complete graph, we present the partition function of the Ising model on an annealed scale-free network as a function of rescaled variables $z$ and $h$. Substituting expansions (60)-(62) for the functions $I_{\lambda}^{ \pm}(x)$ into the partition function (58) one arrives at the following representations: 


$$
Z(t, h)=\left\{\begin{array}{l}
\int_{0}^{+\infty} \exp \left(-t x^{2}-x^{\lambda-1}\right) \cosh (h x) \mathrm{d} x, \quad 3<\lambda<5, \\
\int_{0}^{+\infty} \exp \left(-t x^{2}-x^{4}\right) \cosh (h x) \mathrm{d} x, \quad \lambda \geqslant 5,
\end{array}\right.
$$

where $t$ and $h$ are defined by the equations (67) and (73) respectively.

Figure 15(a) shows results obtained for the motion of the Fisher zeros in the real magnetic field calculated at different $\lambda$ for functions (75). The squares correspond to $\lambda \geqslant 5$ and the expected value of the angle of motion from (76) is $\psi=\pi / 3$. Therefore, in this region of $\lambda$ our numerical results are in a good agreement with the analytical prediction as we get $\psi \simeq 59^{\circ}$. However, this is not the case for the smaller values of $3<\lambda<5$. Indeed, naively substituting critical exponents values into the equation (12) for the angle $\psi$ one gets

$$
\psi=\frac{\pi(\lambda-3)}{2(\lambda-2)}
$$

In particular, from (76) one expects that the angle of motion will decrease with $\lambda$. The three curves from the figure 15(a), calculated for $\lambda=4.8,4.5$ and 4 demonstrate the opposite behaviour: the angle increases with decreasing $\lambda$ with a tendency to reach asymptotics at $\psi=\pi / 2$. As we have shown above, for the model under consideration the zeros at the critical point do not obey the Lee-Yang theorem: there is only a small number of zeros with purely imaginary coordinates for $3<\lambda<5$. Therefore the equation (76) that was obtained under an assumption that this theorem holds is not applicable. The angle $\psi(12)$ describing the motion of Fisher zeros in real magnetic field does not seem to be related by a simple scaling relation to the critical exponents.

Similar to what was done for the Ising model on a complete graph, one can define scaling functions for the real and imaginary parts of the $j$ th zero $z_{j}$. Using the definition (48) of the scaling functions from the section 3.3 for $\mathcal{Z}_{j}^{\mathcal{R}}(h), \mathcal{Z}_{j}^{\mathcal{I}}(h)$ in different regions of $\lambda$ we define the scaling functions for the Ising model on an annealed scale-free network as

$$
\mathcal{Z}_{j}^{R, I}(h) \equiv \begin{cases}\frac{\left\langle k^{2}\right\rangle}{2}[a(\lambda)]^{2 /(\lambda-1)} \mathcal{T}_{j}^{\mathcal{R}, \mathcal{I}}\left(h \frac{\left\langle k^{2}\right\rangle}{\langle k\rangle^{2}} a(\lambda)^{1 /(\lambda-1)}\right), & 3<\lambda<5, \\ \left\langle k^{2}\right\rangle \sqrt{6} / \sqrt{\ln N} \mathcal{T}_{j}^{\mathcal{R}, \mathcal{I}}\left(h \frac{\left\langle k^{2}\right\rangle}{\langle k\rangle^{2}}\left(\frac{\ln N}{24}\right)^{1 / 4}\right), & \lambda=5, \\ \left\langle k^{2}\right\rangle \sqrt{3\left\langle k^{4}\right\rangle} \mathcal{T}_{j}^{\mathcal{R}, \mathcal{I}}\left(h \frac{\left\langle k^{2}\right\rangle}{\langle k\rangle^{2}}\left(\frac{\left\langle k^{4}\right\rangle}{12}\right)^{1 / 4}\right), & \lambda>5,\end{cases}
$$

and expressions for $h$ at different $\lambda$ are given by (73). The scaling functions for the first Fisher zero $z_{1}$ at different values of $\lambda$ are plotted in figure 15(b). From equation (49) we expect that the ratio $\mathcal{Z}_{1}^{I}(0) / \mathcal{Z}_{1}^{R}(0)$ gives the Fisher pinching angle $\varphi$, which in the case of the scale-free network is $\lambda$-dependent (see section 4.2). From figure 15(b) we calculate $\mathcal{Z}_{i}^{I}(0) / \mathcal{Z}_{i}^{R}(0)=0.921 ; 0.798 ; 0.577$ or $\varphi \simeq 0,249 \pi ; 0.228 \pi ; 0.18 \pi$ for $\lambda=4,8 ; 4.5$; 4 respectively. These values agree with those predicted by equation (64) and given in table 2 . Note that the agreement increases with an increase of the zero number $j$.

\section{Conclusions and outlook}

In this paper we analyse the properties of the complex zeros of the partition function for the case of the Ising model on graphs of two types. First, we considered the case of the complete graph and then, we addressed the new features that are introduced by the changes in graph topology, by considering the Ising model on an annealed scale-free network. While the 
former case have been already a subject of several studies (see e.g. [17, 18]), the latter is addressed here for the first time.

In particular, using the approach of [18] we have analysed the behaviour of Lee-Yang zeros as well as tracked the motion of the Fisher zeros in the real external magnetic field. As has been observed before for some other models [5, 28], this motion is governed by the universal angle $\psi$, that encodes values of the order parameter critical exponents through (12). A fundamental feature of the Ising model on the complete graph, as well as of those models where such behaviour of Fisher zeros in real magnetic field was observed, is that their zeros in complex magnetic field at the critical temperature are governed by the Lee-Yang theorem: plotted in the plane $\mathrm{e}^{-h}$ they align along the unit circle [1].

Analysis of section 4 has shown that complex zeros behaviour of the Ising model on an annealed scale-free network is characterized by several new and rather unexpected features. First of all, as far as the universality class of the model is defined by the node-degree distribution decay exponent $\lambda(57)$, the pinching angle $\varphi$ as well as the Lee-Yang edge exponent $\sigma$ are $\lambda$-dependent too. Their dependence on $\lambda$ in the region $3<\lambda<5$ is given by equations (64), (65). In turn, for the fast-decaying degree distributions with $\lambda>5$ we recover the values obtained previously for the Ising model on the complete graph. Secondly, the logarithmic corrections to leading scaling behaviour that arise for the spin models on scalefree networks [33-36] at $\lambda=5$ are observed for the exponents that govern condensation of Lee-Yang and Fisher zeros with an increase of the system size. Corresponding gaps in $H$ - and $t$ planes decrease with $N$ at $\lambda=5$ as

$$
H_{j} \sim N^{-3 / 4}(\ln N)^{1 / 4}, \quad t_{j} \sim N^{-1 / 2}(\ln N)^{1 / 2} .
$$

Note, that the powers of the logarithms comply with the corresponding scaling relations [36].

The striking feature of the Ising model on an annealed scale-free network is that its partition function zeros calculated at $T_{\mathrm{c}}$ in complex magnetic field does not obey the LeeYang theorem. The number of purely imaginary zeros decreases with the decrease of $\lambda$, and the zeros acquire both real and imaginary parts. Note that experimental identification of the Lee-Yang zeros [11] has so far only be made for purely imaginary zeros [10]. The results identified herein show that while these are accessible for lattices and complex networks for sufficiently large $\lambda$, not all network zeros are accessible in this manner for $\lambda<5$. A challenge for experiment is to find another way to access them.

As we have already mentioned, there are some other models, for which the Lee-Yang theorem does not hold [43-54]. Unlike the above examples, the problem we have considered in this paper concerns ferromagnetic Ising model, and the Lee-Yang theorem was proven [56] to hold for any Ising-like model with ferromagnetic interaction, see also [57]. In this respect the analysed here Hamiltonian (50) looks as if it will lead to the Lee-Yang property of the partition function too, since the adjacency matrix elements $J_{l m}$ are non-negative. Furthermore, in the course of partition function calculation, in the spirit of the mean-field approximation, the matrix elements $J_{l m}$ are substituted by $k_{l} k_{m}>0$, see equation (53). Again, this should not spoil the Lee-Yang property for any fixed choice of the node degree $k_{l}$. Next step in the derivation was the integration over $k$ with the distribution function $p(k)$, equation (55). This is the place which leads to the violation of the Lee-Yang unit circle theorem: indeed, if one takes a sum (or an integral) of functions each of which possesses the Lee-Yang property, the sum might not possess such property at all. That is exactly what is observed for the annealed network. 


\section{Acknowledgments}

This work was supported in part by FP7 EU IRSES projects No. 295302 'Statistical Physics in Diverse Realizations' No. 612707 'Dynamics of and in Complex Systems', No. 612669 'Structure and Evolution of Complex Systems with Applications in Physics and Life Sciences' and by the Doctoral College for the Statistical Physics of Complex Systems, LeipzigLorraine-Lviv-Coventry $\left(\mathbb{L}^{4}\right)$. It is our pleasure to thank Yuri Kozitsky, Taras Krokhmalskii, Loïc Turban, and Jean-Yves Fortin for useful comments and discussions. MK is grateful to Taras Hvozd for the help with programming.

\section{Appendix A. Notes on the natural cut-off}

In this appendix we discuss in more detail the choice of the upper cut-off in equation (56). A network of finite size (finite number of nodes $N$ ) cannot contain a node of infinite degree $k$ by definition. For a scale-free network, the maximal node degree $k_{\max }$ for finite $N$ (the so-called natural cut-off) can be defined in different ways. In [58] it was proposed to define a cut-off $k_{\max }$ as the value of the degree for which one expects to observe one vertex at most:

$$
N p\left(k_{\max }\right) \sim 1 \text {. }
$$

From here one gets an $N$ dependency of the cut-off:

$$
k_{\max } \sim N^{1 / \lambda} \text {. }
$$

The drawback of (80) is that for continuous $k$ this definition contains the probability of a single point (which is zero). Another definition of cut-off was given in [30]. There, the cut-off is interpreted as the value of the degree $k$ above which one expects to find at most one vertex:

$$
N \int_{k_{\max }}^{\infty} p(k) \mathrm{d} k \sim 1
$$

leading to

$$
k_{\max } \sim N^{1 /(\lambda-1)} .
$$

In [59] the extreme value theory was used to define the natural cut-off. In this case the exact value for $k_{\max }$ slightly differs, however it remains $N$ dependent. Below we will show that the form of $k_{\max }(N)$ dependence does not change the asymptotic estimate for the partition function (58) in the leading order in $N$.

For simplicity, let us consider the partition function (54) for zero magnetic field $H=0$ (the derivation for non-zero $H$ is quite similar):

$$
Z_{N}(T)=\int_{-\infty}^{+\infty} \exp \left(\frac{-N\langle k\rangle x^{2} T}{2}+\sum_{l} \ln \cosh \left(x k_{l}\right)\right) \mathrm{d} x .
$$

Using (55) we rewrite the sum over network nodes $\sum_{l}$ in the exponent in terms of the integral over $k$ for a given distribution function $p(k)$, keeping the lower and upper cut-off:

$Z_{N}(T)$

$=\int_{-\infty}^{+\infty} \exp \left\{N\left[\frac{-\left\langle k^{2}\right\rangle x^{2} t}{2}+\int_{k_{\min }}^{k_{\max }} p(k)\left[\ln \cosh (x k)-\frac{1}{2}(x k)^{2}\right] \mathrm{d} k\right]\right\} \mathrm{d} x$,

with $t=\left(T-T_{\mathrm{c}}\right) / T_{\mathrm{c}}$ and $T_{\mathrm{c}}=\left\langle k^{2}\right\rangle /\langle k\rangle$. To evaluate (84) we make use of the fact that at large $N$ the main contribution comes from small $x$. In turn, this means that we need to evaluate the integral over $k$ in (84) at small $x$ and for large $N$. To keep the network connected for all 
$3<\lambda<5$ we have chosen the lower cut-off value to be $k_{\min }=2$ (see section 4.1 ). The crucial point is that the value of this integral does not depend on the way in which $k_{\max }$ tends to infinity (e.g. $k_{\max } \sim N^{\frac{1}{\lambda-1}}$ ). It is because $k_{\min }$ and $k_{\max }$ are the only terms in (84) that depend on $N$. Moreover, this dependence may be taken in the form of the natural cut-off (82) or in any other form that satisfies $\lim _{N \rightarrow \infty} k_{\max } \rightarrow \infty$. Therefore, one gets:

$$
\begin{aligned}
& \lim _{x \rightarrow 0 N \rightarrow \infty} \lim _{k_{\min }}^{k_{\max }} p(k)\left[\ln \cosh (x k)-\frac{1}{2}(x k)^{2}\right] \mathrm{d} k \\
& =\lim _{x \rightarrow 0} \int_{2}^{\infty} p(k)\left[\ln \cosh (x k)-\frac{1}{2}(x k)^{2}\right] \mathrm{d} k .
\end{aligned}
$$

The integral in the right-hand side of (85) has been already evaluated (see e.g. [34] or [32]). It is

$$
\lim _{x \rightarrow 0} \int_{2}^{\infty} p(k)\left[\ln \cosh (x k)-\frac{1}{2}(x k)^{2}\right] \mathrm{d} k=a_{\lambda} x^{\lambda-1}+O\left(x^{\lambda}\right),
$$

with known values of the coefficients $a_{\lambda}$ [32, 34]. Substituting (86) into (84) we arrive at:

$$
Z_{N}(\tau) \sim \int_{0}^{\infty} \exp \left\{-N\left(\frac{\left\langle k^{2}\right\rangle x^{2} \tau}{2}+a(\lambda) x^{\lambda-1}\right)\right\} \mathrm{d} x, 3<\lambda<5
$$

Now the integral (87) can be taken by a steepest descent method. The result we have obtained is consistent with the Landau theory for the Ising model on scale-free networks [60] as well as with the mean field analysis [34, 35]. In this way, all three approaches (Landau, mean field, and the current one-based on the integral representation) lead to the same thermodynamics.

\section{Appendix B. Asymptotic estimates for the integral (72)}

In this appendix we determine an asymptotic estimate for the behaviour of the partition function $Z(h)(72)$ at $\operatorname{Re} h=0$ in the limit of large $\operatorname{Im} h$. To this end, let us consider an integral

$$
Z(\mathrm{i} r)=\int_{0}^{\infty} \mathrm{e}^{-x^{\lambda-1}} \cos (r x) \mathrm{d} x
$$

that depends on the real variable $r$. At $r=\operatorname{Im} h$ (88) gives an imaginary part of the partition function (72). Asymptotic behaviour of $Z$ (ir) follows from the Erdelyi lemma (see e.g. [61]) as explained below.

The Erdelyi lemma gives an asymptotic behaviour of the integral:

$$
F(y)=\int_{0}^{A} x^{b-1} f(x) \mathrm{e}^{\mathrm{i} y x^{a}} \mathrm{~d} x
$$

According to the lemma, if $a \geqslant 1, b>0$ and the function $f(x)$ becomes zero together with all its derivatives at the upper integration limit: $f(A)=\left.f^{\prime}(x)\right|_{x=A}=\ldots=\left.f^{(n)}(x)\right|_{x=A}=0$, the following asymptotic estimate for the integral (89) is valid:

$$
F(y) \sim \sum_{k=0}^{\infty} a_{k} y^{-\frac{k+b}{a}}, \quad y \rightarrow \infty
$$


where the coefficients $a_{k}$ are given by

$$
a_{k}=\frac{f^{k}(0)}{k ! a} \Gamma\left(\frac{k+b}{a}\right) \exp \left(\frac{\mathrm{i} \pi(k+b)}{2 a}\right) \text {. }
$$

Changing variables in (88) one can represent $J_{\lambda}(y)$ in the form similar to (90):

$$
Z(\mathrm{i} r)=\frac{5}{\lambda-1} \operatorname{Re} \int_{0}^{\infty} x^{\frac{5}{\lambda-1}-1} \mathrm{e}^{-x^{5}} \mathrm{e}^{\mathrm{i} r x^{5 /(\lambda-1)}} \mathrm{d} x .
$$

Note that the upper integration limit in this case is $a=\infty$. It is easy to see that the conditions of the lemma are satisfied and an asymptotic expansion for the function (88) follows:

$$
Z(\mathrm{i} r) \sim \sum_{k=0}^{\infty} b_{k} r^{-\frac{k(\lambda-1)}{5}-1}, \quad r \rightarrow \infty
$$

where the coefficients $b_{k}$ are defined as

$$
b_{k}=\frac{(\lambda-1) f^{k}(0)}{5 k !} \Gamma\left(\frac{k(\lambda-1)}{5}+1\right) \cos \left(\frac{\pi(k(\lambda-1)+5)}{10}\right) .
$$

Note, that coefficients $b_{k}=0$ for the marginal values $\lambda=3$ and $\lambda=5$ : the decay differs from the power law. To evaluate it, we proceed as follows.

- $\lambda=3$

For $\lambda=3$ the integral (88) is taken exactly leading to

$$
Z(\mathrm{i} r)=\sqrt{\pi / 2} \exp \left(-r^{2} / 4\right) \text {. }
$$

Therefore, in the limit of large $r$ the integral decays exponentially. Moreover, $Z(\mathrm{i} r)>0$ for any $0<r<\infty$ which signals about an absence of partition function zeros on the complex $h$ plane at $\lambda=3$. In turn, this brings about an absence of the phase transition in this case.

- $\lambda=5$

In this case integral (88) can be rewritten as:

$$
Z(\mathrm{i} r)=\int_{0}^{+\infty} \mathrm{e}^{-x^{4}} \cos (r x) \mathrm{d} x=\frac{1}{2} \operatorname{Re} \int_{-\infty}^{+\infty} \mathrm{e}^{-x^{4}} \mathrm{e}^{\mathrm{i} x r} \mathrm{~d} x
$$

Its asymptotics can be evaluated by a steepest descent method, calculating the function under the integral in the point of extremum at $x=(\mathrm{i} r / 4)^{1 / 3}$ and leading to:

$$
Z(\mathrm{i} r) \sim \frac{1}{2} \exp \left(-\frac{3}{2}(r / 4)^{4 / 3}\right) \cos \left(\frac{3 \sqrt{3}}{2}(r / 4)^{4 / 3}\right), \quad r \rightarrow \infty .
$$

The integral Z(ir) has an infinite number of zeros due to the presence of an oscillating function in the rhs, as we further demonstrate below.

In figure 16 we compare behaviour of the numerically calculated function $\log \mid Z$ (ir) | with its asymptotic expansion using equations (93) and (97) for different $\lambda$. One can see that behaviour of the function for $\lambda<5$ is qualitatively different from that at $\lambda \geqslant 5$ : whereas in the last case the function keeps oscillating in the asymptotics (meaning that the number of zeros is unbounded), it is not the case for $3<\lambda<5$. Here, after finite number of oscillations the function approaches its asymptotics from above. Therefore, the number of zeros is limited. As one can see from the figures 16(a), (b), (c), the number of oscillation decreases with the decrease of $\lambda$. 

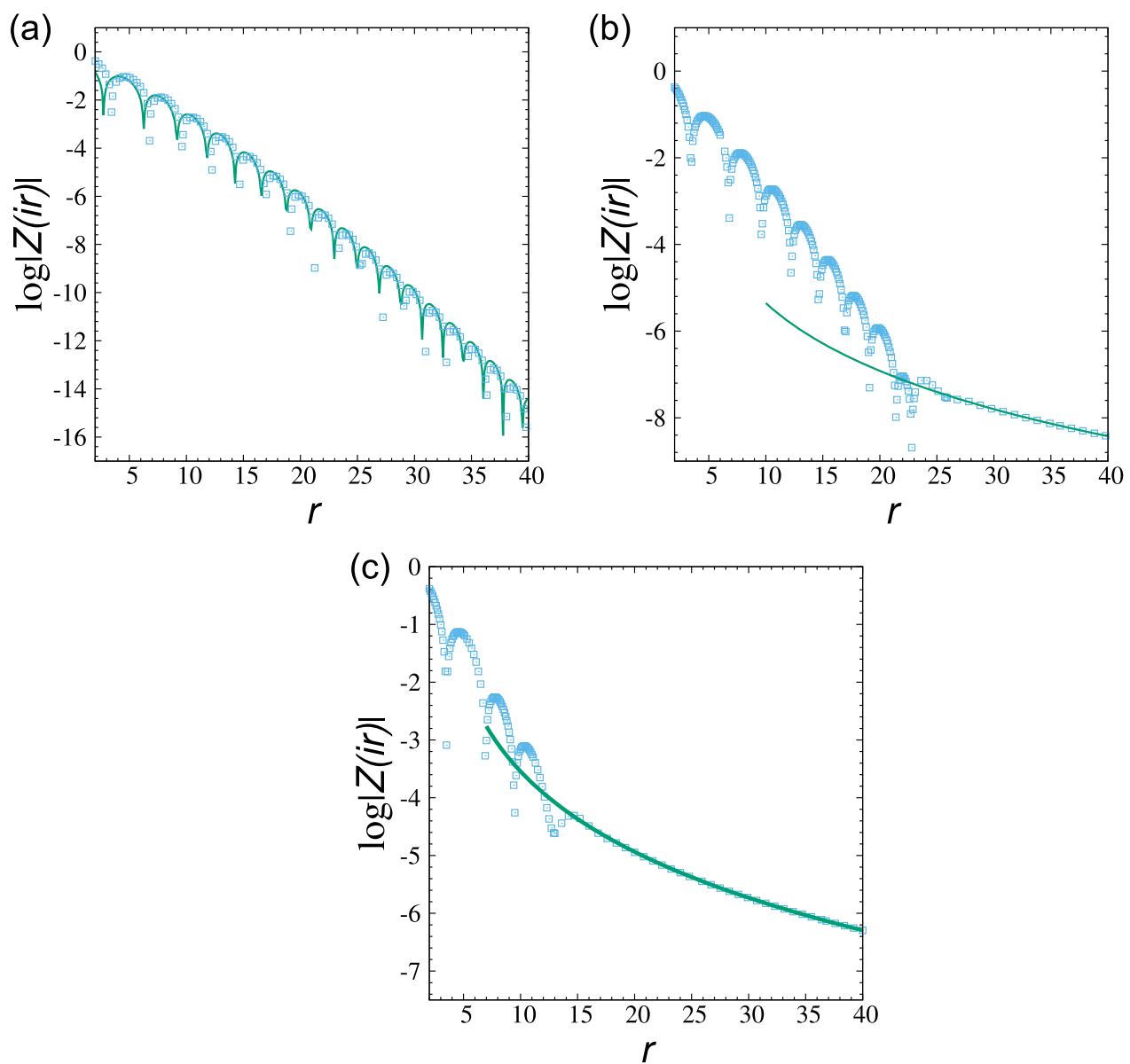

Figure 16. Function $\log \mid Z$ (ir) $\mid$ for: (a) $\lambda \geqslant 5$, (b) $\lambda=4.99$, and (c) $\lambda=4.5$. Solid lines: results of asymptotic expansions. Squares: numerically calculated values. For $\lambda \geqslant 5$ the function keeps oscillating in the asymptotics (meaning that the number of zeros is unbounded), it is not the case for $\lambda<5$.

\section{References}

[1] Yang C N and Lee T D 1952 Phys. Rev. 87404

Yang C N and Lee T D 1952 Phys. Rev. 87410

[2] Fisher M E 1965 The Nature of Critical Points (Lecture Notes in Theoretical Physics vol 7c) (Boulder: University of Colorado Press) pp 1-159

[3] Wu F Y 2008 Int. J. Mod. Phys. B 221899

[4] Pearson R B 1982 Phys. Rev. B 266285

[5] Itzykson C, Pearson R B and Zuber J B 1983 Nucl. Phys. B 220415 [FS8]

[6] Itzykson C and Luck J M 1985 Prog. Phys. 1145

[7] Abe R 1967 Prog. Theor. Phys. 371070 Abe R 1967 Prog. Theor. Phys. 38322

[8] Janke W, Johnston D A and Kenna R 2006 Nucl. Phys. B 736319

[9] Binek C 1998 Phys. Rev. Lett. 815644

Binek C, Kleemann W and Aruga Kotori H 2001 J. Phys.: Condens. Matter 13811

[10] Wei B B and Liu R B 2012 Phys. Rev. Lett. 109185701

[11] Peng X, Zhou H, Wei B B, Cui J, Du J and Liu R B 2015 Phys. Rev. Lett. 114010601 
[12] Albert R and Barabasi A L 2002 Mod. Phys. Rev. 7447

Dorogovtsev S N and Mendes J F F 2003 Evolution of Networks: From Biological Networks to the Internet and $W W W$ (Oxford: Oxford University Press)

Holovatch Y, von Ferber C, Olemskoi A, Holovatch T, Mryglod O, Olemskoi I and Palchykov V 2006 J. Phys. Stud. 10247 (in Ukrainian)

Barrat A, Barthelemy M and Vespignani A 2008 Dynamical Processes on Complex Networks (Cambridge: Cambridge University Press)

Newman M 2010 Networks: An Introduction (Oxford: Oxford University Press)

[13] Dorogovtsev S N, Goltsev A V and Mendes J F F 2008 Rev. Mod. Phys. 801275

[14] Lee S H, Ha M, Jeong H, Noh J D and Park H 2009 Phys. Rev. E 80051127

[15] Brout R 1959 Phys. Rev. 115824

[16] Bianconi G 2012 Phys. Rev. E 85061113

[17] Dommers S, Giardinà C, Giberti $C$, van der Hofstad R and Prioriello M L arXiv:1509.07327v1 Giardinà C, Giberti C, van der Hofstad R and Prioriello M L arXiv:1509.02695v1

[18] Glasser M L, Privman V and Schulman L S 1986 J. Stat. Phys. 451841

[19] Glumac Z and Uzelac K 2013 Phys. Rev. E 66022140

[20] Krasnytska M, Berche B, Holovatch Y and Kenna R 2015 EPL 11160009

[21] Glasser M L, Privman V and Schulman L S 1987 Phys. Rev. B 351841

[22] Kac M 1968 Mathematical Mechanisms of Phase Transitions Statistical Physics: Phase Transitions and Superfluidity ed M Chretien et al (New York: Gordon and Breach) pp 241-305

[23] Stanley H E 1971 Introduction to Phase Transitions and Critical Phenomena (Oxford and New York: Oxford University Press)

[24] Gradshteyn I S and Ryzhik I M 2014 Table of Integrals, Series, and Products 8th edn ed D Zwillinger and V Moll (New York: Academic) p 1184

[25] Janke W and Kenna R 2001 J. Stat. Phys. 1021211

[26] Baños R A, Gil-Narvion J M, Monforte-Garcia J, Ruiz- Lorenzo J J and Yllanes D 2013 J. Stat. Mech. P02031

[27] Gordillo-Guerrero A, Kenna R and Ruiz-Lorenzo J J 2013 Phys. Rev. E 88062117

[28] Zuber J B 1982 Non-perturbative Field Theory and QCD: Proc. (Trieste) pp 228-241 SACLAYSPH-T-83-004 CEA-CONF-6639

[29] Privman V 1990 Finite Size Scaling and Numerical Simulation of Statistical Systems (World Scientific: Singapore)

Privman V, Hohenberg P C and Aharony A 1991 Phase Transitions and Critical Phenomena ed $\mathrm{C}$ Domb and J L Lebowitz vol 14 (New York: Academic)

[30] Dorogovtsev S N, Goltsev A V and Mendes J F F 2002 Phys. Rev. E 66016104

[31] Aiello W, Park F and Lu L 2000 Proc. STOC'O0 Proc. 32th annual ACM Symp. on Theory of computing (New York: American Mathematical Society) p 171

[32] Krasnytska M, Berche B and Holovatch Y 2013 Condens. Matter Phys. 1623602

[33] Palchykov V, von Ferber C, Folk R, Holovatch Y and Kenna R 2010 Phys. Rev. E 82011145

[34] Igloi F and Turban L 2002 Phys. Rev. E 66036140

[35] Leone M, Vázquez A, Vespignani A and Zecchina R 2002 Eur. Phys. J. B 28191 Dorogovtsev S, Goltsev A V and Mendes J F F 2004 Eur. Phys. J. B 38177

von-Ferber C, Folk R, Holovatch Y, Kenna R and Palchykov V 2011 Phys. Rev. E 83061114

[36] Kenna R 2013 Order, Disorder and Criticality ed Y Holovatch vol 3 (Singapore: World Scientific) pp $1-46$

[37] Dunlop F and Newman C M 1975 Commun. Math. Phys. 44223

[38] Simon B and Griffiths R B 1973 Commun. Math. Phys. 33145

[39] Wang X Z 2001 Phys. Rev. E 63046103

Wang X Z 2007 Physica A 380163

[40] Blythe R A and Evans M R 2002 Phys. Rev. Lett. 89080601

[41] O'Loan O J, Evans M R and Cates M E 1998 Phys. Rev. E 581404

[42] Chou T and Lohse D 1999 Phys. Rev. Lett. 823552

[43] Kim S Y 2004 Phys. Rev. Lett. 93130604

[44] Kim S Y 2005 Nucl. Phys. B 705504 [FS]

[45] Suzuki M 1973 J. Math. Phys. 141088

[46] Yamada K, Nakano H and Yamashita M 1981 Prog. Theor. Phys. 651603

[47] Monroe J L 1991 J. Stat. Phys. 65445 
[48] Chin K K and Landau D P 1987 Phys. Rev. B 36275

[49] Nilsen T S 1967 Physica 3747

[50] Hemmer P C and Hiis Hauge E 1964 Phys. Rev. 133 A1010

[51] Lee K C 1994 Phys. Rev. Lett. 732801

[52] Biskup M, Borgs C, Chayes J T, Kleinwaks L J and Kotecký R 2000 Phys. Rev. Lett. 844794

[53] Wang X Z and Kim J S 1998 Phys. Rev. E 584174

[54] Tong P and Liu X 2006 Phys. Rev. Lett. 97017201

[55] Bena I, Droz M and Lipowski A 2005 Int. J. Mod. Phys. B 194269

[56] Lieb E H and Sokal A D 1981 Commun. Math. Phys. 80153

[57] Kozitsky Y 1997 J. Stat. Phys. 87799

Kozitsky Y 2003 Appl. Math. Comput. 141103

[58] Aiello W, Chung F and Lu L 2001 Exp. Math. 1053

[59] Boguna M, Pastor-Satorras R and Vespignani A 2004 Eur. Phys. J. B 38205

[60] Goltsev A V, Dorogovtsev S N and Mendes J F F 2003 Phys. Rev. E 67026123

[61] Fedoryuk M V 1989 Asymptotic Methods in Analysis (Analysis I Encyclopaedia of Mathematical Sciences vol 13) ed R V Gamkrelidze (Berlin: Springer) pp 83-191 